\title{
Species Traits Drive Long-Term Population Trends of Common Breeding Birds in Northern Italy
}

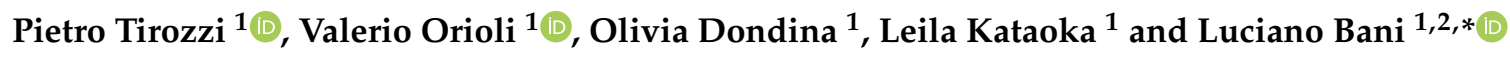 \\ 1 Department of Earth and Environmental Sciences, University of Milano-Bicocca, Piazza della Scienza 1, \\ 20126 Milano, Italy; p.tirozzi@campus.unimib.it (P.T.); valerio.orioli@unimib.it (V.O.); \\ olivia.dondina@unimib.it (O.D.); 1.kataoka@campus.unimib.it (L.K.) \\ 2 World Biodiversity Association Onlus c/o NAT LAB Forte Inglese, Portoferraio, 57037 Livorno, Italy \\ * Correspondence: luciano.bani@unimib.it
}

check for updates

Citation: Tirozzi, P.; Orioli, V.; Dondina, O.; Kataoka, L.; Bani, L. Species Traits Drive Long-Term Population Trends of Common Breeding Birds in Northern Italy. Animals 2021, 11, 3426. https:// doi.org/10.3390/ani11123426

Academic Editor: Jukka Jokimäki

Received: 11 October 2021

Accepted: 29 November 2021

Published: 1 December 2021

Publisher's Note: MDPI stays neutral with regard to jurisdictional claims in published maps and institutional affiliations.

Copyright: (C) 2021 by the authors. Licensee MDPI, Basel, Switzerland. This article is an open access article distributed under the terms and conditions of the Creative Commons Attribution (CC BY) license (https:/ / creativecommons.org/licenses/by/ $4.0 /)$.
Simple Summary: We assessed population trends for breeding birds in Lombardy (N Italy) from 1992 to 2019 and investigated the relationships between the observed trends and groups of species sharing similar characteristics (i.e., functional groups). We found a general positive or stable situation for $76 \%$ of the species. However, about $24 \%$ of the species declined, with worrying negative trends (greater than $-50 \%$ ) for two-thirds of them. Regarding species groups, we found that populations of migrants, of species with short incubation period, and of species with high annual fecundity declined. Similarly, populations of plant-eaters, of species feeding on invertebrates, and of farmland birds decreased. Only populations of woodland birds increased. In conclusion, our study provided a portrait of the status of common breeding birds in the region. Moreover, by analyzing the population response of the functional groups, we identified which of them experienced the most significant population changes, providing the foundations to implement studies aimed at quantifying the effects of specific divers responsible of the observed population changes in these groups.

\begin{abstract}
Long-term population trends are considerable sources of information to set wildlife conservation priorities and to evaluate the performance of management actions. In addition, trends observed in functional groups (e.g., trophic guilds) can provide the foundation to test specific hypotheses about the drivers of the observed population dynamics. The aims of this study were to assess population trends of breeding birds in Lombardy (N Italy) from 1992 to 2019 and to explore the relationships between trends and species sharing similar ecological and life history traits. Trends were quantified and tested for significance by weighted linear regression models and using yearly population indices (median and 95\% confidence interval) predicted through generalized additive models. Results showed that $45 \%$ of the species increased, $24 \%$ decreased, and $31 \%$ showed nonsignificant trends. Life history traits analyses revealed a general decrease of migrants, of species with short incubation period and of species with high annual fecundity. Ecological traits analyses showed that plant-eaters and species feeding on invertebrates, farmland birds, and ground-nesters declined, while woodland birds increased. Further studies should focus on investigation of the relationship between long-term trends and species traits at large spatial scales, and on quantifying the effects of specific drivers across multiple functional groups.
\end{abstract}

Keywords: functional groups; life history; ecological traits; generalized additive models; population trend; birds

\section{Introduction}

Wildlife monitoring programs are fundamental to implement species conservation strategies and to verify to what extent they are effective [1-3]. Population trends represent one of the most informative proxy of the status of populations' health, from local to continental spatial scales. One of the most monitored taxa worldwide are birds $[4,5]$, 
an animal group of large conservation interest, target of several conservation policies in different countries, as well as at a continental scale (e.g., Birds Directive 2009/147/EC in European Union). From the past century, many bird monitoring programs have been started in many countries worldwide, both at a continental scale (e.g., the Pan-European Common Bird Monitoring Scheme-PECBMS - in Europe [6], the North American Breeding Bird Survey-BBS-[7]) and at a national scale (e.g., in Finland [8,9], the Breeding Bird Survey-BBS - in UK [10]). In other cases, many data is available, although these have often been collected within projects with a specific purpose other than long-term monitoring [11]. Historical data collected in different years by different projects are surely a valuable but often overlooked source of information, available for the evaluation of the conservation status of species, communities, and ecosystems [12-15]. Beyond the assessment of population dynamic of each bird species, which is essential to define species-specific conservation priorities and actions, birds represent valuable ecological indicators that can potentially provide useful information about the overall biodiversity and ecosystem processes [16-20]. Changes in species assemblages can be driven by species-specific responses to environmental perturbations (either natural or anthropic), and identifying which factors cause the observed changes is crucial to mitigate the impact of these perturbations. Although species population trends are not directly informative on drivers of population changes [21], observing a common trend among species with similar ecological or life history traits can provide the foundation to test specific hypotheses about the drivers of the observed population dynamics [22]. Despite the arising attention of a trait-based approach in community ecology [15,23-29] and population ecology [30-34], only in the last few years researchers have started to explore the link between traits and population trends in avifauna over long time periods, highlighting the existence of different patterns among distinct functional groups [22,35-41].

In Europe, the decline of long-distance migrants wintering in Sub-Saharan Africa compared to short-distance migrants or sedentary species has been emphasized in several studies (e.g., ref. [22,35,42]), as well as the general decline of farmland birds, as underlined by the tendency of the Common Farmland Birds Indicator [6]. Gregory et al. [43] highlighted the existence, in the period from 1980 to 2015 and across 28 European countries, of different trends among farmland species and generalist forest species, with the first ones decreasing and the second ones stable. However, some contrasting trends were found for old forest species due to forest management [9]. Insectivorous birds are declining in Europe [37]. In Germany, however, this trend has not been confirmed for the entire guild, since differences emerged between trends of farmland insectivorous birds and forest insectivorous birds, with the former decreasing and the latter stable [22]. Moreover, species with narrow niche breadth (i.e., specialist species) may suffer more from human-driven environmental alterations than species with broad niche breadth (i.e., generalist species), resulting in contrasting trends between the two groups $[40,44]$. Nesting location can influence the breeding success by affecting the exposure of the nest to predation (e.g., birds or mammals, [45]). For example, ground-nesting bird populations have decreased during recent decades in Europe $[46,47]$ with most dramatic declines reported for larger species such as waders and bustards inhabiting agricultural landscape $[48,49]$ or forest grouses (Tetraonidae, $[50,51])$. In addition, many others less explored life history traits (e.g., incubation and fledging period, body mass, fecundity, species' mobility) can provide useful insights about the relationship between population trends and groups of species sharing similar traits.

In Italy, where a long-term monitoring program at national scale is available only from 2000 (MITO2000 projects, [52,53]), no studies explored this relationship over long time series. However, in Lombardy (a region in the north of Italy), bird data from different projects have been collected for almost 30 years (1992-ongoing), and they can be merged to assess long-term population trends in the region, while accounting for different sources of data heterogeneity (i.e., environmental bias, overdispersion and zero-inflation, [54], see Section 2.3). In this study, we aimed at estimating trends for common breeding birds in Lombardy from 
1992 to 2019, and at investigating how species traits are linked to population trends, in order to identify which species need of major conservation attention and which are the functional groups that are experiencing the most relevant demographic changes.

\section{Materials and Methods}

\subsection{Study Area}

The study was carried out in Lombardy, a region of $23,861 \mathrm{~km}^{2}$ in Northern Italy $\left(45^{\circ} \mathrm{N}, 9^{\circ} \mathrm{E}\right)$. The northern part of the area is characterized by mountains Alps and Prealps, separated from each other by the wide glacial valley of Valtellina. The southwestern corner is characterized by the hilly landscape of northern Apennines. The rest of the region hosts a large portion of the alluvial plain of the Po River (the widest plain in Italy) extending from west to east for more than $12,000 \mathrm{~km}^{2}$. The average elevation of the whole region is $610 \mathrm{~m}$ above sea level (a.s.l.), but it shows a large variation across different areas, ranging from $2 \mathrm{~m}$ a.s.l. of the plain to roughly $4000 \mathrm{~m}$ a.s.l. of the Alps. Land use is characterized by urban areas $(14.7 \%)$, agricultural lands $(42.2 \%)$, and natural and semi-natural lands $(39.6 \%$, of which $61.4 \%$ are forests). Alps and Prealps are mainly characterized by coniferous forests, meadows and grasslands at higher elevations, and deciduous forest at lower ones. The Apennine area is characterized by vineyards, extensive farming and deciduous and mixed forests. The Po Plain is heavily men-modified, with intensive cereal cultivations (mainly maize) in the central and eastern part, and dense urban areas and paddy fields in the west (Figure 1).

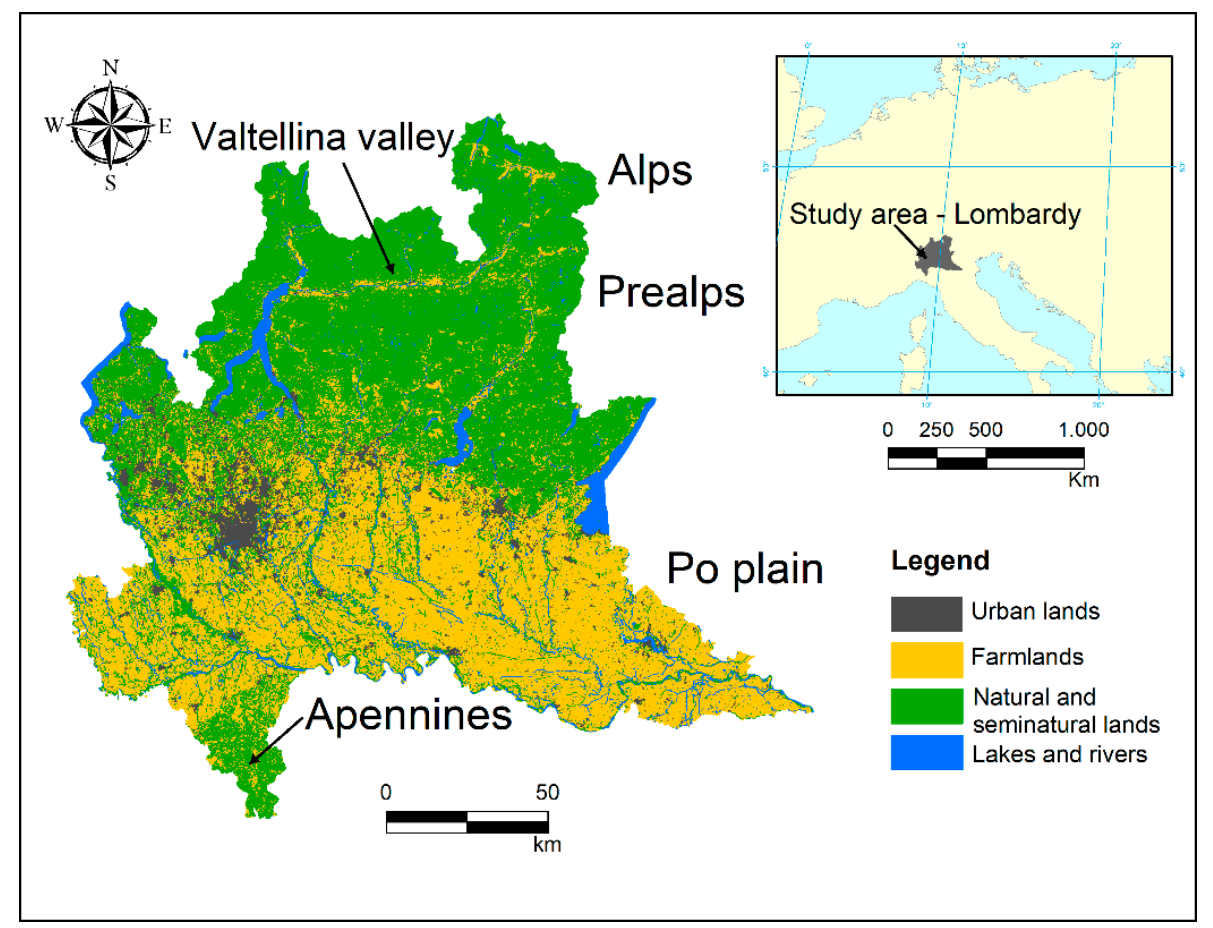

Figure 1. Study area. Land use refers to DUSAF 2018 digital map, level 1 of classification [55].

\subsection{Survey Design and Bird Data}

Data were collected from five different projects on breeding bird surveys in Lombardy carried out from 1992 to 2019. Overall, the dataset consists of 18,505 point counts, with an average of 771 point counts per year (range 373-1443; SD = 244). No data are available for years 1993, 1994, 1997, and 1998 (Table S1). The first project was the "Long-term Monitoring Program", launched in 1992 and covering 18 years. It consisted in a stratified sampling design, where seven primary sampling units were defined based on the main landscapes present in the Region. Within each primary sampling units, secondary sampling units, corresponding to about $10 \mathrm{~km} \times 10 \mathrm{~km}$ squares ("Tavolette IGMI", 1:25,000 maps) 
were extracted proportionally to the representativeness of each landscape, and yearly renewed to guarantee complete coverage of the Region in the long-term. Point counts were randomly located within secondary sampling units, according to territory accessibility. From 2007 to 2016, fixed secondary sampling units (20 in 2007, 21 in 2008, 23 in 2009-2010, 24 in 2011-2016) were added to the random ones. Finally, starting from 2017, all sampling units became fixed (34 units per year, including all of them previously performed). The "Regional Fauna Database" project was carried out from 2000 to 2006, using a systematic stratified sampling design. Finally, other three projects ("Forest Biodiversity Survey", "Lowland Biodiversity Survey", and "Greenway Project") were performed in restricted subareas of the region during a limited period (few years) with the aims of surveying breeding birds living in forests, in agricultural lands and in the Apennines, respectively (Table S2). In all projects, data were collected using a single-visit point-count method with unlimited distance [56]. The minimum distance between sampling locations was $500 \mathrm{~m}$ within each year. All birds heard or seen in 10 min were recorded [57]. Bird surveys were performed during the breeding season (10 May to 20 June) to minimize the count of migrants (birds not breeding in the study area) and to survey territorial birds. Surveys were conducted from sunrise to 11.00 a.m., only in good weather conditions, sunny to cloudy, without rain or strong wind [58]. This technique provides a measure of relative bird abundance $[59,60]$. The technique is effective in detecting bird species belonging to the orders Columbiformes, Cuculiformes, Apodiformes, Coraciiformes, Piciformes, and Passeriformes [58], but can also be used to survey some other common species, such as the Common Buzzard (Buteo buteo) and the Common Kestrel (Falco tinnunculus). All counts were expressed in number of breeding pairs according to Blondel et al. [56]. For early-breeding sedentary species (e.g., tits) that in our study area might start to breed in February-March, the sampling period might not entirely fit. Actually, this may represent a weakness in the detection of such species. However, since the early-breeding sedentary species can be detected in groups composed by parents and offspring during the late spring, each family group was converted into one breeding pairs in order to not overestimate the counts. Although some limitations can arise for some species starting to breed in the early spring (e.g., woodpeckers), the survey window we set (10 May to 20 June) can be considered suitable for most of species that can be detected by point counts technique in our study area. Additionally, even if an underestimate of detected individuals whose territorially behaviour peak precedes the beginning of the survey period, this source of bias remain constant over years. This way, counts of early-breeding sedentary species do not affect the trend estimates. In the case of large-scale monitoring programs, it is essential to obtain an optimal trade-off between costs (i.e., within-season multiple surveys effective to detect early- and late-breeders) and benefits (i.e., large representative sample). Thus, we prefer to obtain a large representative sample, relying on single-visit survey, accepting an underestimation of early-breeders that do not jeopardize the trend estimates.

Since data did not rely on within-season multiple surveys, it was impossible to account for species detection probability. However, although the detection probability might represent an issue to be addressed in trend analysis relying on density estimates [61], while using relative abundance data coming from a large dataset, where the effect of stochasticity on species detection is limited [25], accounting for the detection probability can be considered superfluous (Appendix A). Among the 204 species detected in the whole period, we modelled trends for those with an overall relative frequency higher than $2 \%$.

To avoid an overestimation of the number of breeding pairs for gregarious species during the breeding season, whose groups are composed of parental pairs and fledglings, we used the following conversion factor (CF) (i.e., the number of individuals considered as one breeding pair [62]): Feral Pigeon (Columba livia domestica), CF = 8; Long-tailed Tit (Aegithalos caudatus), CF = 14; Common Starling (Sturnus vulgaris), CF = 11; Italian Sparrow (Passer italiae) and House Sparrow (Passer domesticus), CF = 14; Eurasian Tree Sparrow (Passer montanus), $\mathrm{CF}=16$. For instance, in the case of the Common Starling, the detection 
from one to 11 individuals has been converted into one breeding pairs, from 12 to 22 individuals into two breeding pairs, and so on.

\subsection{Species Trend Assessment}

Our dataset is affected by environmental bias inherited from spatial bias (i.e., the inadequate representation of the variability of environmental covariates in the study area $[63,64])$, due to differences in the sampling design of the five projects merged to obtain the final dataset. This issue prevents the trend assessment by using original data [54]. Furthermore, overdispersion (i.e., the variance is larger than the mean) and zero-inflation (i.e., a particular type of overdispersion due to an excess of zero counts) could be present in count data [65-67], and ignoring them can lead to serious errors in the interpretation of results from an ecological perspective [68]. To handle environmental bias, overdispersion and zero-inflation, we adopted the modelling procedure described in [54], which is summarized in the following part of the Section and to which the reader can refer for a more detailed explanation. In their work, authors assessed long-term population trends for six bird species adopting a full-factorial design to account for environmental bias, overdispersion and zero-inflation. Since environmental bias resulted the most relevant factor to determine the magnitude of trend estimates, in our analyses we excluded models not accounting for that. Following the aforementioned approach, we performed, starting from the original count data, four generalized additive models (GAMs [69-71]), namely C-ZIP-GAM, C-ZINB-GAM, C-P-GAM, C-NB-GAM (C, model with covariates dealing with environmental bias; ZIP, zero-inflated Poisson; ZINB, zero-inflated negative binomial; P, Poisson; NB, negative binomial). GAMs allow for relaxing parametric assumptions of generalised linear models (GLMs [72]), replacing some, or all, of the parametric terms by smooth functions of the covariates. We used the year of survey as a parametric factor; 17 land cover variables (continue urban matrix and infrastructures, discontinue urban matrix, arable lands, paddy fields, vineyards, orchards, olive groves, wood plantations, meadows and pastures, broadleaved forests, mixed forests, coniferous forests, grasslands, shrub lands, areas with sparse or absent vegetation, wetland vegetation, rivers and streams) recorded as percentage cover within a $250 \mathrm{~m}$ circular buffer around each survey site and four topographic variables (average values within a $250 \mathrm{~m}$ circular buffer around each survey site of elevation, sine and cosine of the aspect, slope) as covariates to account for environmental bias; and the spatial trend (interactions between X and Y coordinates (UTM 32N, WGS84 Datum)) to account for spatial pattern in the data [73] and potential spatial autocorrelation [12,25]. For the zero-inflated GAMs, elevation and the percentage cover of urban and forest area within a $2500 \mathrm{~m}$ circular buffer designed around the survey site were used as predictors to explain the zero-inflation process. All covariates were modelled as smooth function (maximum degree of freedom set at four), except for the sine and cosine of the aspect which were modelled as linear effect. Based on the AIC [74], for each species, the best of the four models was picked out. Thus, it was used to predict yearly population indices through a parametric bootstrap with 1000 simulations [75]. We used the median of the distribution of bootstrapped predictions as population index estimator for each year, and calculated the $95 \%$ confidence intervals by the percentile method [76]. Land cover values in the prediction matrix were predicted from GAMs wherein the yearly average values for each covariate, derived from the six-digital land-use vector map DUSAF available from 1980 to 2019 (DUSAF 1980, 1999, 2007, 2012, 2015, and 2018, downloadable from http: / / www.geoportale.regione.lombardia.it/, accessed on 21 September 2021) were fitted as smooth function of year. In addition, since topographic variables and coordinates are time-invariant, they were fixed at the overall mean and the centroid of the area, respectively. Since some species could have clustered or geographically restricted distribution (e.g., alpine species), we used point counts in which the species was present in the period 1992-2019 to determine the species distribution area $\left(\mathrm{km}^{2}\right)$ across the region (Minimum Convex Hull method, or $\mathrm{MCH}$ ). For species that had a ratio between the $\mathrm{MCH}$ and the 
regional area less than 0.80 , we used the $\mathrm{MCH}$ as polygon to extract values for all covariates; otherwise, the entire Region was used.

To assess the long-term population trends, a weighted least square linear regression (WLS) was performed, using the median of the yearly population index as response variable (estimated number of breeding pairs per sampling site), the year as continuous explanatory variable, and the reciprocal of the width of the confidence interval associated to the yearly estimate as weight. We acknowledge that yearly indices are not temporally independent, but since our aim was to assess long-term trends, a linear regression can be considered adequate for the purpose [77,78]. The model can be summarized as $I_{t}=\beta \times Y e a r+\varepsilon_{i}$, $w=1 / C I$, whereby we tested whether the trend was significantly different from zero $(\mathrm{p}(\beta) \leq 0.05)$. The variation in population dimension from 1992 to $2019(\mathrm{~T} \%)$ was quantified as $T \%=\left[\left(I_{2019}-I_{1992}\right) / I_{1992}\right] \times 100$.

Geospatial analyses were performed in ESRI ArcMap 10.7.1 (Redlands, CA, USA), while trend analyses in R software [79] using the packages mcgv [80] and zigam [81]. Since these last analyses were particularly resource-consuming, they were performed on the supercomputer Marconi-A3 HPC [82].

\subsection{Trait-Based Analysis}

\subsubsection{Bird Traits}

To explore whether species sharing similar life history and ecological traits showed a similar demographic signal in response to specific drivers, we selected a set of 12 traits (Table S3). After evaluating the association among each pair of traits (categorical variables) through the Cramer's V coefficient [83] using R package $v c d$ [84]), we retained those traits not significantly associated with the others (Chi-Square test of independence or Fisher's exact test for expected frequencies less than five). In case of significant relationships, we retained only traits with a weak or moderate degree of association (Cramer's V $<0.5[85,86]$; Figure S1). After this check, four life history and four ecological traits were selected for the following analyses. Life history traits encompassed the migration strategy, dispersal ratio, annual fecundity, and incubation period. Migration strategy was derived from [87], which reports detailed information about phenology of populations in the study area. We classified species into three groups: "sedentary species" (non-migrants), "short-distance migrants" (wintering in Europe or North Africa), and "long-distance migrants" (wintering in Sub-Saharan Africa). Dispersal ratio (mean wing length [mm]/cube root of mean body mass [g]) was used as index of species' mobility [88]. Annual fecundity was calculated as the product of average clutch size and average number of broods per year [89], and incubation period was the mean duration of eggs' incubation (days). We derived these information from [90]. For the Common Cuckoo (Cuculus canorus), we set to one the number of average broods per year. We transformed dispersal ratio, annual fecundity and incubation period from numerical to categorical variables, following the approach suggested by [22]. We used the first quartile of the variable values of all species considered in the analyses (Dispersal ratio: values $\leq 28.278$, Annual fecundity: values $\leq 5$, Incubation period: values $\leq 13$ ) to identify the groups "low dispersal ratio", "low annual fecundity", and "short incubation period". The upper quartile (Dispersal ratio: values $\geq 33.737$, Annual fecundity: values $\geq 10.625$, Incubation period: values $\geq 17.25$ ) was used to define the levels "high dispersal ratio", "high annual fecundity", and "long incubation period". Finally, the values in-between were considered to represent species with intermediate characteristics. Ecological traits included diet, nest type, landscape type, and degree of specialization. Diet represents the main source of food on which the species feeds on. It was derived from information collected in [91], where the percentage of food items used by the species is reported. We assigned species to the level "vertebrates" if it feeds on at least $70 \%$ of vertebrates, to "plant-eaters" if it feeds on at least $70 \%$ of vegetal material (seeds, nectar, fruits, other part of plants), and to "invertebrates" if it eats at least $70 \%$ of invertebrates (arthropods, mollusks, annelids). We assigned species to "omnivores" whether none of the foregoing categories individually exceeded the threshold of $70 \%$. 
The Black Kite (Milvus migrans), resulting as scavenger for at least 70\%, was classified as "vertebrates". Nest type represents the nest position and it was derived from [90]; we reclassified the original categories "open-arboreal" and "closed-arboreal" into the group "elevated-nesters", "ground" and "ground-closer" into "ground-nesters", and finally "hole-nesters" were retained as in the original study. Common Cuckoo was assigned to "elevated-nesters" [92]. Landscape type represents the habitat preference of the species at landscape scale. To derive this trait we used our dataset that, being of large dimension and temporal coverage, can be considered a reliable source of information on habitat usage by the species. We calculated the median of fractional cover for each level (urban, agricultural, forest, natural open-habitat, wetlands-rivers-lakes, classified according to DUSAF map [55]) within a radius of $2500 \mathrm{~m}$ around the point counts where the species was present. Point counts of each year were related to the temporally closest available DUSAF or CORINE [93] digital map. Whether the median of the fractional cover of a specific level was greater than $50 \%$ we assigned that level as landscape type, namely "farmland", "woodland", and "natural open-habitat" (such as shrubs, grasslands, rocks). Whether the median did not exceed $50 \%$ for any levels we assigned the category "several". To calculate the degree of specialization we started from information collected in [92]. We calculated single-species specialization indices [94] for each of the five following traits: food type, acquisition behavior, substrate from which food is acquired, foraging habitats, nesting habitats. Subsequently, we derived an overall specialization index (SI) by computing the mean of the five indices. Hence, the higher the SI, the greater the species specialization. In foraging habitats, we added "urban" and "garden" as habitats used by the Feral Pigeon, and "dry grassland", "urban" and "garden" by the Eurasian Magpie (Pica pica). Moreover, in the substrate from which food is acquired category, we grouped the levels "watersurface", "underwater" and "water" into a single level.

\subsubsection{Relationship between Population Indices and Traits}

To test whether species belonging to the same functional group showed similar responses in terms of population trends, and if differences among trends of different groups were significant, we used a distinct weighted linear regression model for each trait. The median of the yearly population index was considered as response variable, "year", the trait and their interaction as explanatory variables, and the reciprocal of the width of the confidence interval associated to each median yearly index was used as weight. The interaction term represents the trend for the specific group. In order to test whether the coefficient for each interaction term was significantly different from zero and from the other levels, we carried out additional statistic tests using the finite-sample F statistic by the function linearHypothesis of the R package car [95].

As the absolute value of the population index is not directly comparable among different species since it depends on the regional species abundance, we divided, within each species, the median of the yearly index by the median value of the index in the first year of the time series (i.e., $\mathrm{I}_{1992}$ ). Exceptions were made for the Great Cormorant (Phalacrocorax carbo) ( $\left.\mathrm{I}_{2005}\right)$, Northern Lapwing (Vanellus vanellus), Short-toed Treecreeper (Certhia brachydactyla) and Common Linnet (Linaria cannabina) $\left(\mathrm{I}_{1995}\right)$ since they were not recorded in 1992. To obtain comparable confidence intervals (used as weights in the model), we adopted the identical procedure for the 2.5th and the 97.5th percentiles. In this way, we assigned the value one at the median population index in the first year, and all the others indices (median, 2.5th and 97.5th percentiles) were expressed as variation in relation to the median of the first year.

\section{Results}

\subsection{Species Population Trends}

We assessed population indices and trends for 76 breeding bird species. According to AIC ranking, C-P-GAM (Poisson GAM with covariates dealing with environmental bias) was picked out as best model for 12 species, C-NB-GAM (negative binomial GAM with 
covariates dealing with environmental bias) for nine, C-ZIP-GAM (zero-inflated Poisson GAM with covariates dealing with environmental bias) for 40, and C-ZINB-GAM-zeroinflated negative binomial GAM with covariates dealing with environmental bias-for 15 (Table S4). In the selected models (see Table S5 for models' summary), the explained deviance ranged between $10.90 \%$ and $77.80 \%$ (mean $=38.61 \%)$ in the non-zero-inflated GAMs, while it ranged between $52.90 \%$ and $97.20 \%$ (mean $=82.62 \%$ ) and between $7.30 \%$ and $66.60 \%$ (mean $=30.27 \%$ ) in the binomial (i.e., zero-inflated part) and count component of the zero-inflated GAMs, respectively (Table S4). Long-term trend analyses resulting from the weighted least square linear regression (WLS), highlighted a significant positive trend $(\mathrm{T} \%)$ for 34 species (ranging from $+12,060 \%$ for the Great Cormorant to $+14.45 \%$ for the Eurasian Blackcap), negative for 18 species, 12 of which experienced a population decrease larger than $50 \%$, while 24 species did not show a significant trend ( $p$ value $>0.05$; Table 1 and Figure S2).

Table 1. Summary of weighted least square linear regression (WLS) for each species. Column "WLS model" indicates the best model selected by AIC, used to perform the linear regression. $\mathrm{C}$, model with covariates dealing with environmental bias; ZIP, zero-inflated Poisson; ZINB, zero-inflated negative binomial; P, Poisson; NB, negative binomial; GAM, generalized additive model. $\beta$, estimate of regression coefficient for the explanatory variable "Year"; SE, standard error of $\beta$; $t$-value, $t$ statistic; T\% 1992-2019, percentage of change in the population dimension from 1992 to 2019 according to the WLS model (significant trends are marked in bold); Adj- $\mathrm{R}^{2}$ : adjusted R-square (in case of negative value it was round to zero). Estimates and standard errors for the intercept are not showed. The common names of the species are presented according to the nomenclature suggested by [96].

\begin{tabular}{|c|c|c|c|c|c|c|c|}
\hline Species & WLS Model & $\beta^{1}$ & $\mathrm{SE}^{1}$ & t Value & $p$ Value & $\begin{array}{c}\mathrm{T} \% \\
1992-2019\end{array}$ & Adj- $R^{2}$ \\
\hline $\begin{array}{c}\text { Great Cormorant } \\
\text { (Phalacrocorax carbo) }\end{array}$ & C-NB-GAM & 0.050 & 0.007 & -7.046 & $<0.001$ & $+12,060$ & 0.78 \\
\hline $\begin{array}{l}\text { Black-crowned Night Heron } \\
\text { (Nycticorax nycticorax) }\end{array}$ & C-NB-GAM & -0.112 & 0.068 & -1.661 & 0.111 & -47.86 & 0.07 \\
\hline $\begin{array}{c}\text { Little Egret } \\
\text { (Egretta garzetta) }\end{array}$ & C-NB-GAM & 0.366 & 0.134 & 2.745 & 0.012 & +100.59 & 0.22 \\
\hline $\begin{array}{l}\text { Grey Heron } \\
\text { (Ardea cinerea) }\end{array}$ & C-NB-GAM & 0.022 & 0.008 & 2.719 & 0.013 & +54.76 & 0.22 \\
\hline $\begin{array}{c}\text { Mallard } \\
\text { (Anas platyrhynchos) }\end{array}$ & C-ZINB-GAM & 0.019 & 0.003 & 6.776 & $<0.001$ & +525.19 & 0.66 \\
\hline $\begin{array}{c}\text { Black Kite } \\
\text { (Milous migrans) }\end{array}$ & C-ZINB-GAM & -0.053 & 0.022 & -2.459 & 0.022 & -54.96 & 0.18 \\
\hline $\begin{array}{l}\text { Common Buzzard } \\
\text { (Buteo buteo) }\end{array}$ & C-P-GAM & 0.074 & 0.052 & 1.416 & 0.171 & +50.42 & 0.04 \\
\hline $\begin{array}{l}\text { Common Kestrel } \\
\text { (Falcon tinnunculus) }\end{array}$ & C-P-GAM & 0.083 & 0.018 & 4.760 & $<0.001$ & +239.37 & 0.49 \\
\hline $\begin{array}{l}\text { Common Quail } \\
\text { (Coturnix coturnix) }\end{array}$ & C-ZIP-GAM & -0.044 & 0.030 & -1.471 & 0.155 & -46.83 & 0.05 \\
\hline $\begin{array}{l}\text { Common Pheasant } \\
\text { (Phasianus colchicus) }\end{array}$ & C-NB-GAM & 0.121 & 0.016 & 7.596 & $<0.001$ & +591.31 & 0.71 \\
\hline $\begin{array}{l}\text { Common Moohren } \\
\text { (Gallinula chloropus) }\end{array}$ & C-NB-GAM & -0.008 & 0.009 & -0.819 & 0.421 & -13.83 & 0 \\
\hline $\begin{array}{l}\text { Northern Lapwing } \\
\text { (Vanellus vanellus) }\end{array}$ & C-NB-GAM & 0.218 & 0.033 & 6.514 & $<0.001$ & +1653.42 & 0.65 \\
\hline $\begin{array}{c}\text { Feral Pigeon } \\
\text { (Columba livia domestica) }\end{array}$ & C-ZINB-GAM & 0.062 & 0.079 & 0.779 & 0.444 & +30.28 & 0 \\
\hline $\begin{array}{l}\text { Common Wood Pigeon } \\
\text { (Columba palumbus) }\end{array}$ & C-ZIP-GAM & 1.213 & 0.175 & 6.945 & $<0.001$ & +1727.33 & 0.67 \\
\hline
\end{tabular}


Table 1. Cont.

\begin{tabular}{|c|c|c|c|c|c|c|c|}
\hline Species & WLS Model & $\beta^{1}$ & $\mathrm{SE}^{1}$ & t Value & $p$ Value & $\begin{array}{c}\mathrm{T} \% \\
1992-2019 \\
\end{array}$ & Adj- $R^{2}$ \\
\hline $\begin{array}{c}\text { Eurasian Collared Dove } \\
\text { (Streptopelia decaocto) }\end{array}$ & C-ZINB-GAM & 0.415 & 0.060 & 6.876 & $<0.001$ & +196.25 & 0.67 \\
\hline $\begin{array}{l}\text { European Turtle Dove } \\
\text { (Streptopelia turtur) }\end{array}$ & C-ZIP-GAM & 0.253 & 0.279 & 0.907 & 0.374 & +22.53 & 0 \\
\hline $\begin{array}{l}\text { Common Cuckoo } \\
\text { (Cuculus canorus) }\end{array}$ & C-ZIP-GAM & 0.104 & 0.227 & 0.458 & 0.651 & +5.81 & 0 \\
\hline $\begin{array}{l}\text { Common Swift } \\
\text { (Apus apus) }\end{array}$ & C-ZINB-GAM & -1.857 & 0.583 & -3.187 & 0.004 & -50.01 & 0.29 \\
\hline $\begin{array}{l}\text { European Bee-eater } \\
\text { (Merops apiaster) }\end{array}$ & C-NB-GAM & 0.287 & 0.045 & 6.367 & $<0.001$ & +1325.78 & 0.63 \\
\hline $\begin{array}{l}\text { Eurasian Wryneck } \\
\text { (Jinx torquilla) }\end{array}$ & C-ZIP-GAM & -0.484 & 0.074 & -6.557 & $<0.001$ & -81.59 & 0.65 \\
\hline $\begin{array}{l}\text { European Green } \\
\text { Woodpecker } \\
\text { (Picus viridis) }\end{array}$ & C-P-GAM & 0.316 & 0.043 & 7.314 & $<0.001$ & +418.48 & 0.70 \\
\hline $\begin{array}{l}\text { Great Spotted Woodpecker } \\
\text { (Dendrocopos major) }\end{array}$ & C-P-GAM & 0.425 & 0.034 & 12.448 & $<0.001$ & +1530.25 & 0.87 \\
\hline $\begin{array}{l}\text { Eurasian Skylark } \\
\text { (Alauda arvensis) }\end{array}$ & C-ZIP-GAM & -0.744 & 0.087 & -8.521 & $<0.001$ & -99.65 & 0.76 \\
\hline $\begin{array}{l}\text { Eurasian Crag Martin } \\
\text { (Ptyonoprogne rupestris) }\end{array}$ & C-NB-GAM & 0.078 & 0.052 & 1.498 & 0.148 & +64.74 & 0.05 \\
\hline $\begin{array}{l}\text { Barn Swallow } \\
\text { (Hirundo rustica) }\end{array}$ & C-ZINB-GAM & -1.788 & 0.288 & -6.209 & $<0.001$ & -67.41 & 0.62 \\
\hline $\begin{array}{l}\text { Common House Martin } \\
\text { (Delichon urbicum })\end{array}$ & C-ZINB-GAM & -0.864 & 0.276 & -3.127 & 0.005 & -45.55 & 0.28 \\
\hline $\begin{array}{c}\text { Tree Pipit } \\
\text { (Anthus trivialis) }\end{array}$ & C-ZIP-GAM & 0.134 & 0.176 & 0.761 & 0.455 & +14.41 & 0 \\
\hline $\begin{array}{c}\text { Water Pipit } \\
\text { (Anthus spinoletta) }\end{array}$ & C-ZINB-GAM & 0.019 & 0.025 & 0.746 & 0.463 & +15.12 & 0 \\
\hline $\begin{array}{l}\text { Western Yellow Wagtail } \\
\text { (Motacilla flava) }\end{array}$ & C-ZIP-GAM & -1.057 & 0.149 & -7.073 & $<0.001$ & -64.79 & 0.68 \\
\hline $\begin{array}{c}\text { Grey Wagtail } \\
\text { (Motacilla cinerea) }\end{array}$ & C-P-GAM & 0.003 & 0.034 & 0.088 & 0.931 & +1.36 & 0 \\
\hline $\begin{array}{l}\text { White Wagtail } \\
\text { (Motacilla alba) }\end{array}$ & C-P-GAM & -0.338 & 0.126 & -2.693 & 0.013 & -33.56 & 0.21 \\
\hline $\begin{array}{c}\text { Eurasian Wren } \\
\text { (Troglodytes troglodytes) }\end{array}$ & C-ZIP-GAM & -0.054 & 0.087 & -0.626 & 0.538 & -8.91 & 0 \\
\hline $\begin{array}{c}\text { Dunnock } \\
\text { (Prunella modularis) }\end{array}$ & C-P-GAM & 0.597 & 0.101 & 5.923 & $<0.001$ & +309.03 & 0.60 \\
\hline $\begin{array}{l}\text { European Robin } \\
\text { (Erithacus rubecula) }\end{array}$ & C-ZIP-GAM & 0.140 & 0.053 & 2.640 & 0.015 & +35.51 & 0.21 \\
\hline $\begin{array}{l}\text { Common Nigthingale } \\
\text { (Luscinia megarhynchos) }\end{array}$ & C-ZIP-GAM & -0.356 & 0.116 & -3.055 & 0.006 & -35.32 & 0.27 \\
\hline $\begin{array}{c}\text { Black Redstart } \\
\text { (Phoenicurus ochruros) }\end{array}$ & C-ZIP-GAM & 0.161 & 0.021 & 7.523 & $<0.001$ & +135.77 & 0.71 \\
\hline $\begin{array}{c}\text { Common Redstart } \\
\text { (Phoenicurus Phoenicurus) }\end{array}$ & C-ZIP-GAM & 0.569 & 0.121 & 4.682 & $<0.001$ & +126.60 & 0.48 \\
\hline
\end{tabular}


Table 1. Cont.

\begin{tabular}{|c|c|c|c|c|c|c|c|}
\hline Species & WLS Model & $\beta^{1}$ & $\mathrm{SE}^{1}$ & t Value & $p$ Value & $\begin{array}{c}\mathrm{T} \% \\
1992-2019\end{array}$ & Adj- $R^{2}$ \\
\hline $\begin{array}{l}\text { African Stonechat } \\
\text { (Saxicola torquatus) }\end{array}$ & C-ZIP-GAM & -0.687 & 0.143 & -4.793 & $<0.001$ & -87.83 & 0.49 \\
\hline $\begin{array}{l}\text { Northern Wheatear } \\
\text { (Oenanthe oenanthe) }\end{array}$ & C-P-GAM & -0.001 & 0.006 & -0.136 & 0.893 & -3.67 & 0 \\
\hline $\begin{array}{l}\text { Common Blackbird } \\
\text { (Turdus merula) }\end{array}$ & C-ZIP-GAM & 1.291 & 0.682 & 1.894 & 0.072 & +25.15 & 0.10 \\
\hline $\begin{array}{l}\text { Song Thrush } \\
\text { (Turdus philomelos) }\end{array}$ & C-ZIP-GAM & 0.561 & 0.051 & 11.026 & $<0.001$ & +2869.64 & 0.84 \\
\hline $\begin{array}{l}\text { Mistle Trush } \\
\text { (Turdus viscivorus) }\end{array}$ & C-ZIP-GAM & 0.439 & 0.078 & 5.608 & $<0.001$ & +468.08 & 0.57 \\
\hline $\begin{array}{l}\text { Cetti's Warbler } \\
\text { (Cettia cetti) }\end{array}$ & C-ZIP-GAM & -0.096 & 0.031 & -3.049 & 0.006 & -62.35 & 0.27 \\
\hline $\begin{array}{l}\text { Melodius Warbler } \\
\text { (Hippolais polyglotta) }\end{array}$ & C-ZIP-GAM & 0.273 & 0.044 & 6.154 & $<0.001$ & +346.07 & 0.62 \\
\hline $\begin{array}{l}\text { Lesser Whitethroat } \\
\text { (Curruca curruca) }\end{array}$ & C-ZIP-GAM & 0.141 & 0.042 & 3.320 & 0.003 & +153.05 & 0.30 \\
\hline $\begin{array}{l}\text { Eurasian Blackcap } \\
\text { (Sylvia atricapilla) }\end{array}$ & C-ZIP-GAM & 0.848 & 0.387 & 2.192 & 0.039 & +14.50 & 0.14 \\
\hline $\begin{array}{l}\text { Western Bonelli's Warbler } \\
\quad(\text { Phylloscopus bonelli) }\end{array}$ & C-ZINB-GAM & 0.355 & 0.110 & 3.214 & 0.004 & +79.62 & 0.29 \\
\hline $\begin{array}{l}\text { Common Chiffchaff } \\
\text { (Phylloscopus collybita) }\end{array}$ & C-ZIP-GAM & -0.154 & 0.053 & -2.919 & 0.008 & -40.64 & 0.25 \\
\hline $\begin{array}{c}\text { Goldcrest } \\
\text { (Regulus regulus) }\end{array}$ & C-ZIP-GAM & -0.102 & 0.047 & -2.184 & 0.040 & -41.42 & 0.14 \\
\hline $\begin{array}{l}\text { Common Firecrest } \\
\text { (Regulus ignicapilla) }\end{array}$ & C-ZIP-GAM & 0.363 & 0.084 & 4.349 & $<0.001$ & +309.79 & 0.44 \\
\hline $\begin{array}{l}\text { Spotted Flycatcher } \\
\text { (Muscicapa striata) }\end{array}$ & C-P-GAM & 1.477 & 0.209 & 7.080 & $<0.001$ & +490.70 & 0.68 \\
\hline $\begin{array}{c}\text { Long-tailed Tit } \\
\text { (Aegithalos caudatus) }\end{array}$ & C-P-GAM & 0.186 & 0.049 & 3.781 & 0.001 & +114.79 & 0.37 \\
\hline $\begin{array}{c}\text { Marsh Tit } \\
\text { (Poecile palustris) }\end{array}$ & C-ZIP-GAM & 0.295 & 0.049 & 6.006 & $<0.001$ & +340.76 & 0.60 \\
\hline $\begin{array}{c}\text { Willow Tit } \\
\text { (Poecile montanus) }\end{array}$ & C-ZIP-GAM & 0.155 & 0.068 & 2.289 & 0.032 & +116.63 & 0.16 \\
\hline $\begin{array}{l}\text { European Crested Tit } \\
\text { (Lophophanes cristatus) }\end{array}$ & C-ZIP-GAM & 0.072 & 0.020 & 3.640 & 0.001 & +141.11 & 0.35 \\
\hline $\begin{array}{c}\text { Coal Tit } \\
\text { (Periparus ater) }\end{array}$ & C-ZIP-GAM & 0.046 & 0.040 & 1.155 & 0.261 & +20.66 & 0.01 \\
\hline $\begin{array}{l}\text { Eurasian Blue Tit } \\
\text { (Cyanistes caeruleus }\end{array}$ & C-ZIP-GAM & 0.179 & 0.071 & 2.538 & 0.019 & +48.78 & 0.19 \\
\hline $\begin{array}{c}\text { Great Tit } \\
\text { (Parus major) }\end{array}$ & C-ZIP-GAM & 1.615 & 0.248 & 6.502 & $<0.001$ & +104.37 & 0.64 \\
\hline $\begin{array}{l}\text { Eurasian Nuthatch } \\
\quad \text { (Sitta europea) }\end{array}$ & C-ZIP-GAM & 0.015 & 0.009 & 1.788 & 0.088 & +81.84 & 0.09 \\
\hline $\begin{array}{l}\text { Short-toed Treecreeper } \\
\text { (Certhia brachydactyla) }\end{array}$ & C-ZIP-GAM & 0.160 & 0.022 & 7.155 & $<0.001$ & +998.50 & 0.70 \\
\hline $\begin{array}{l}\text { Eurasian Golden Oriole } \\
\quad \text { (Oriolus oriolus) }\end{array}$ & C-ZIP-GAM & 0.229 & 0.067 & 3.404 & 0.003 & +75.75 & 0.32 \\
\hline
\end{tabular}


Table 1. Cont.

\begin{tabular}{|c|c|c|c|c|c|c|c|}
\hline Species & WLS Model & $\beta^{1}$ & $\mathrm{SE}^{1}$ & t Value & $p$ Value & $\begin{array}{c}\mathrm{T} \% \\
1992-2019\end{array}$ & Adj-R ${ }^{2}$ \\
\hline $\begin{array}{l}\text { Red-backed Shrike } \\
\text { (Lanius collurio) }\end{array}$ & C-P-GAM & -0.576 & 0.112 & -5.128 & $<0.001$ & -80.13 & 0.52 \\
\hline $\begin{array}{c}\text { Eurasian Jay } \\
\text { (Garrulus glandarius) }\end{array}$ & C-P-GAM & 0.146 & 0.020 & 7.218 & $<0.001$ & +174.93 & 0.69 \\
\hline $\begin{array}{l}\text { Eurasian Magpie } \\
\quad \text { (Pica pica) }\end{array}$ & C-ZIP-GAM & 0.279 & 0.029 & 9.541 & $<0.001$ & +753.86 & 0.80 \\
\hline $\begin{array}{l}\text { Carrion Crow } \\
\text { (Corvus corone) }\end{array}$ & C-ZIP-GAM & -0.008 & 0.044 & -0.192 & 0.850 & -6.24 & 0 \\
\hline $\begin{array}{l}\text { Hooded Crow } \\
\text { (Corvus cornix) }\end{array}$ & C-ZINB-GAM & 0.395 & 0.301 & 1.309 & 0.204 & +13.31 & 0.03 \\
\hline $\begin{array}{l}\text { Common Starling } \\
\text { (Sturnus vulgaris) }\end{array}$ & C-ZINB-GAM & -0.261 & 0.177 & -1.473 & 0.155 & -18.55 & 0.05 \\
\hline $\begin{array}{l}\text { Italian Sparrow } \\
\text { (Passer italiae) }\end{array}$ & C-ZIP-GAM & -1.902 & 0.235 & -8.077 & $<0.001$ & -71.06 & 0.74 \\
\hline $\begin{array}{c}\text { Eurasian Tree Sparrow } \\
\text { (Passer montanus) }\end{array}$ & C-ZIP-GAM & -0.272 & 0.064 & -4.253 & $<0.001$ & -41.31 & 0.43 \\
\hline $\begin{array}{l}\text { Common Chaffinch } \\
\text { (Fringilla coelebs) }\end{array}$ & C-ZIP-GAM & -0.333 & 0.286 & -1.161 & 0.258 & -5.45 & 0.02 \\
\hline $\begin{array}{l}\text { European Serin } \\
\text { (Serinus serinus) }\end{array}$ & C-ZIP-GAM & -0.111 & 0.345 & -0.322 & 0.751 & -4.49 & 0 \\
\hline $\begin{array}{l}\text { European Greenfinch } \\
\text { (Chloris chloris) }\end{array}$ & C-ZINB-GAM & -2.590 & 0.338 & -7.657 & $<0.001$ & -82.00 & 0.72 \\
\hline $\begin{array}{c}\text { European Goldfinch } \\
\text { (Carduelis carduelis) }\end{array}$ & C-ZINB-GAM & -2.477 & 0.297 & -8.329 & $<0.001$ & -86.89 & 0.75 \\
\hline $\begin{array}{l}\text { Common Linnet } \\
\text { (Linaria cannabina) }\end{array}$ & C-ZINB-GAM & 0.034 & 0.044 & 0.767 & 0.452 & +20.60 & 0 \\
\hline $\begin{array}{l}\text { Common Redpoll } \\
\text { (Acanthis flammea) }\end{array}$ & C-ZINB-GAM & -0.427 & 0.247 & -1.727 & 0.098 & -42.55 & 0.08 \\
\hline $\begin{array}{l}\text { Eurasian Bullfinch } \\
\text { (Pyrrhula pyrrhula) }\end{array}$ & C-ZIP-GAM & 0.099 & 0.063 & 1.561 & 0.133 & +73.22 & 0.06 \\
\hline
\end{tabular}

${ }^{1}$ Values are raised to $10^{2}$ to ease comparisons.

3.2. Relation between Population Trends and Species Traits

3.2.1. Life History Traits

Results for life-history traits were summarized in Table 2A-D. Sedentary species showed an increasing but not significant trend, while short and long-distance migrants significantly decreased (Figure 2A), but the magnitude of decline did not differ significantly between these two groups (Table $2 \mathrm{~A}, p$ value $=0.517$ ). Species with intermediate dispersal ratio showed a significant decreasing trend (Figure $2 \mathrm{~B}$ ), even if it was not significantly different from the trend estimated for species with high and low dispersal ratio (Table 2B, $p$ value $=0.194$ and $p$ value $=0.295$, respectively). Analyses for the annual fecundity showed that species with high annual fecundity decreased (Figure 2C), and their decline was significantly different from the (negative but non-significant) trends of the other two groups (Table 2C). Finally, concerning the incubation period, only species with low incubation period showed a significant trend, which was negative (Table 2D and Figure 2D). 
Table 2. Weighted linear regression models showing the response of avian functional groups in relation to life-history traits. In "model output" are shown model statistics (estimate, estimated coefficient; SE, standard error; $t$ value, $t$-statistic) and the significance of each coefficient ( $p$ value) The term "Intercept" and "Year" represent the reference group. The number of species included in each group is shown in parentheses. In "Additional tests" are shown tests of equivalence to zero of interaction effect of non-reference levels and tests of equivalence between the interaction terms for the other levels (F value: F statistic). Significant $p$ values $(\leq 0.05)$ for the interaction terms are marked in bold (A) Migration strategy. Groups: sedentary (reference level), short distance migrants (SDM), long distance migrants (LDM); adj- $\mathrm{R}^{2}=0.05$. (B) Dispersal ratio: Groups: high dispersal ratio (reference level), intermediate dispersal ratio (IDR), limited dispersal ratio (LDR); $\operatorname{adj}^{-} \mathrm{R}^{2}=0.01$. (C) Annual fecundity. Groups: high annual fecundity (reference level), intermediate annual fecundity (IAF), low annual fecundity (LAF); adj- $\mathrm{R}^{2}=0.02$. (D) Incubation period. Groups: short incubation period (reference level), intermediate incubation period (IIP), long incubation period (LIP); adj- $R^{2}=0.04$.

\begin{tabular}{|c|c|c|c|c|}
\hline \multicolumn{5}{|c|}{ (A) Migration Strategy } \\
\hline \multicolumn{5}{|c|}{ Model output } \\
\hline Term & Estimate & SE & t Value & $p$ Value \\
\hline Intercept (25) & -14.441 & 9.176 & -1.574 & 0.116 \\
\hline SDM (30) & 40.904 & 11.808 & 3.464 & $<0.001$ \\
\hline LDM (21) & 33.821 & 12.351 & 2.738 & 0.006 \\
\hline Year & 0.008 & 0.005 & 1.683 & 0.093 \\
\hline Year: SDM & -0.021 & 0.006 & -3.486 & $<0.001$ \\
\hline Year: LDM & -0.017 & 0.006 & -2.769 & 0.006 \\
\hline \multicolumn{5}{|c|}{ Additional tests } \\
\hline Null hypothesis & & & F Value & $p$ Value \\
\hline Year: $\mathrm{SDM}=0$ & & & 11.966 & $<0.001$ \\
\hline Year: $\mathrm{LDM}=0$ & & & 5.143 & 0.023 \\
\hline $\begin{array}{l}\text { Year: SDM = Year: } \\
\text { LDM }\end{array}$ & & & 0.391 & 0.532 \\
\hline \multicolumn{5}{|c|}{ (B) Dispersal ratio } \\
\hline \multicolumn{5}{|c|}{ Model output } \\
\hline Term & Estimate & SE & t Value & $p$ Value \\
\hline Intercept (19) & 7.039 & 9.335 & 0.754 & 0.451 \\
\hline IDR (38) & 15.051 & 11.582 & 1.300 & 0.194 \\
\hline LDR (19) & 2.484 & 13.642 & 0.182 & 0.856 \\
\hline Year & -0.003 & 0.005 & -0.672 & 0.502 \\
\hline Year: IDR & -0.008 & 0.006 & -1.300 & 0.194 \\
\hline Year: LDR & -0.001 & 0.007 & -0.176 & 0.860 \\
\hline \multicolumn{5}{|c|}{ Additional tests } \\
\hline Null hypothesis & & & F Value & $p$ Value \\
\hline Year: IDR $=0$ & & & 9.683 & 0.002 \\
\hline Year: LDR $=0$ & & & 0.761 & 0.383 \\
\hline Year: IDR= Year: LDR & & & 1.097 & 0.295 \\
\hline \multicolumn{5}{|c|}{ (C) Annual fecundity } \\
\hline \multicolumn{5}{|c|}{ Model output } \\
\hline Term & Estimate & SE & t Value & $p$ Value \\
\hline Intercept (19) & 33.284 & 9.141 & 3.641 & $<0.001$ \\
\hline IAF (37) & -24.554 & 11.909 & -2.062 & 0.039 \\
\hline LAF (20) & -29.050 & 12.367 & -2.349 & 0.019 \\
\hline Year & -0.016 & 0.005 & -3.570 & $<0.001$ \\
\hline Year: IAF & 0.012 & 0.006 & 2.083 & 0.037 \\
\hline Year: LAF & 0.015 & 0.006 & 2.357 & 0.019 \\
\hline
\end{tabular}


Table 2. Cont.

\begin{tabular}{|c|c|c|c|c|}
\hline \multicolumn{5}{|c|}{ (C) Annual fecundity } \\
\hline \multicolumn{5}{|c|}{ Additional tests } \\
\hline Null hypothesis & & & F Value & $p$ Value \\
\hline Year: $\mathrm{IAF}=0$ & & & 1.051 & 0.305 \\
\hline Year: $\mathrm{LAF}=0$ & & & 0.174 & 0.676 \\
\hline Year: IAF = Year: LAF & & & 0.148 & 0.701 \\
\hline \multicolumn{5}{|c|}{ (D) Incubation period } \\
\hline \multicolumn{5}{|c|}{ Model output } \\
\hline Term & Estimate & SE & t Value & $p$ Value \\
\hline Intercept (29) & 25.684 & 6.411 & 4.006 & $<0.001$ \\
\hline $\operatorname{IIP}(28)$ & -23.327 & 11.436 & -2.040 & 0.042 \\
\hline LIP (19) & -33.426 & 12.549 & -2.664 & 0.008 \\
\hline Year & -0.012 & 0.003 & -3.902 & $<0.001$ \\
\hline Year: IIP & 0.012 & 0.006 & 2.063 & 0.039 \\
\hline Year: LIP & 0.017 & 0.006 & 2.682 & 0.007 \\
\hline \multicolumn{5}{|c|}{ Additional tests } \\
\hline Null hypothesis & & & F Value & $p$ Value \\
\hline Year: IIP $=0$ & & & 0.022 & 0.881 \\
\hline Year: LIP $=0$ & & & 0.643 & 0.423 \\
\hline Year: IIP= Year:LIP & & & 0.492 & 0.483 \\
\hline
\end{tabular}

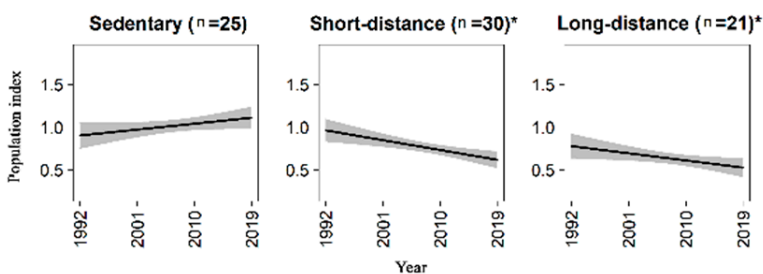

(A)

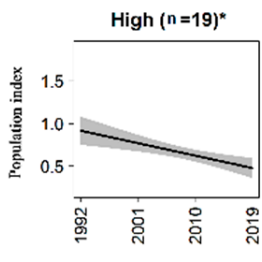

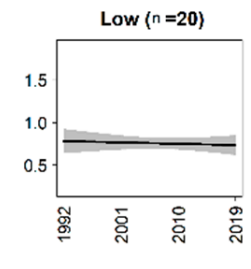

(C)
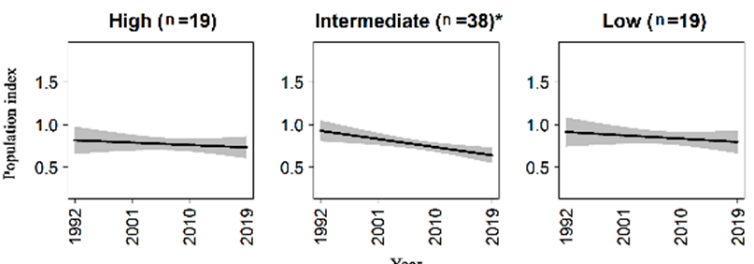

(B)
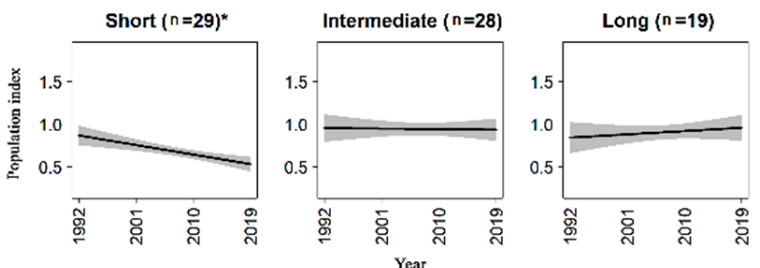

(D)

Figure 2. Population trends for bird species grouped according to the life history traits. (A) Migration strategy; (B) dispersal ratio; (C) annual fecundity; (D) incubation period. Y-axis represents the population index of the species (see Section 2.4.2 for details). Shaded area represents the $95 \%$ confidence interval of the regression line. The number of species in each group is shown in parentheses. The asterisk indicates the significance of the trend in relation to non-zero slope.

\subsubsection{Ecological Traits}

Results for ecological traits were summarized in Table 3A-D. As regards the diet, pairwise comparison among groups did not show significant differences. However, tests for a non-zero slope within each group revealed a significant decline for plant-eaters and species feeding on invertebrates, while omnivores and species eating vertebrates did not exhibit any significant trend (Table 3A and Figure 3A). Ground-nesters showed a decreasing trend, while neither elevated-nesters nor hole-nesters resulted to have significant population changes (Table 3B and Figure 3B). Species inhabiting farmland landscapes showed a clear decrease, while species preferring woodland landscapes resulted to be on 
increase (Figure 3C). Although species of natural open-habitat showed a raising tendency, the trend was not significant, maybe due to the limited sample dimension for this group (nine species). Finally, for the group of species classified as "several" (i.e., those not having a prevalent habitat preference) the regression coefficient for the interaction term was roughly equal to zero (Table 3C). In relation to the overall specialization index, the model highlighted a significant negative trend for species with a medium degree of specialization, while both specialists and generalists did not show significant population changes over the period (Figure 3D). However, no differences were detected among trends of the three groups, very probably due to the weak dissimilarity among the coefficients (Table 3D).

Table 3. Weighted linear regression models showing the response of avian functional groups in relation to ecological traits. In "model output" are shown model statistics (Estimate, estimated coefficient; SE, standard error; $t$ value, $t$-statistic) and the significance of each coefficient ( $p$ value) The term "Intercept" and "Year" represent the reference group. The number of species included in each group is shown in parentheses. In "Additional tests" are shown tests of equivalence to zero of interaction effect of non-reference levels and tests of equivalence between the interaction terms for the other levels (F value: F statistic). Significant $p$ values $(\leq 0.05)$ for the interaction terms are marked in bold. (A) Diet. Groups: omnivores (references level), plant-eaters (PLA), invertebrates (INV), vertebrates (VER); adj-R ${ }^{2}=0.01$. (B) Nest type. Groups: elevated-nesters (reference level), groundnesters (GR), hole-nesters (HL); adj- $R^{2}=0.02$. (C) Landscape type. Groups: open natural habitat (reference level), farmland (FAR), woodland (WOO), several (SEV); adj-R ${ }^{2}=0.11$. (D) SI. Groups: generalist species (reference level), intermediate species (INT), specialist species (SPE); adj- $\mathrm{R}^{2}=0.02$.

\begin{tabular}{|c|c|c|c|c|}
\hline \multicolumn{5}{|c|}{ (A) Diet } \\
\hline \multicolumn{5}{|c|}{ Model output } \\
\hline Term & Estimate & SE & t Value & $p$ Value \\
\hline Intercept (31) & 6.242 & 7.250 & 0.861 & 0.389 \\
\hline $\operatorname{PLA}(13)$ & 22.686 & 12.764 & 1.777 & 0.076 \\
\hline INV (26) & 12.264 & 11.541 & 1.063 & 0.288 \\
\hline VER (6) & -0.662 & 20.755 & -0.032 & 0.975 \\
\hline Year & -0.003 & 0.004 & -0.743 & 0.458 \\
\hline Year: PLA & -0.011 & 0.006 & -1.791 & 0.074 \\
\hline Year: INV & -0.006 & 0.006 & -1.069 & 0.285 \\
\hline Year: VER & 0.000 & 0.010 & 0.021 & 0.983 \\
\hline \multicolumn{5}{|c|}{ Additional tests } \\
\hline Null hypothesis & & & F Value & $p$ Value \\
\hline Year: PLA $=0$ & & & 7.232 & 0.007 \\
\hline Year: INV $=0$ & & & 3.898 & 0.048 \\
\hline Year: VER $=0$ & & & 0.065 & 0.799 \\
\hline Year: PLA = Year:INV & & & 0.579 & 0.446 \\
\hline Year: PLA = Year: VER & & & 1.111 & 0.292 \\
\hline Year: INV = Year: VER & & & 0.356 & 0.551 \\
\hline \multicolumn{5}{|c|}{ (B) Nest type } \\
\hline \multicolumn{5}{|c|}{ Model output } \\
\hline Term & Estimate & SE & t Value & $p$ Value \\
\hline Intercept (35) & 5.627 & 6.834 & 0.823 & 0.410 \\
\hline GR (13) & 29.582 & 13.532 & 2.186 & 0.029 \\
\hline HL (28) & 9.696 & 10.731 & 0.904 & 0.366 \\
\hline Year & -0.002 & 0.003 & -0.703 & 0.482 \\
\hline Year: GR & -0.015 & 0.007 & -2.203 & 0.028 \\
\hline Year: HL & -0.005 & 0.005 & -0.903 & 0.367 \\
\hline
\end{tabular}


Table 3. Cont.

\begin{tabular}{|c|c|c|c|c|}
\hline \multicolumn{5}{|c|}{ (B) Nest type } \\
\hline \multicolumn{5}{|c|}{ Additional tests } \\
\hline Null hypothesis & & & F Value & $p$ Value \\
\hline Year: GR = 0 & & & 8.790 & 0.003 \\
\hline Year: HOLE = 0 & & & 3.070 & 0.080 \\
\hline Year: GR = Year: HL & & & 1.976 & 0.160 \\
\hline \multicolumn{5}{|c|}{ (C) Landscape type } \\
\hline \multicolumn{5}{|c|}{ Model output } \\
\hline Term & Estimate & SE & t Value & $p$ Value \\
\hline Intercept (9) & -22.340 & 20.764 & -1.076 & 0.282 \\
\hline FAR (31) & 50.341 & 21.718 & 2.318 & 0.021 \\
\hline WOO (17) & -14.078 & 26.297 & -0.535 & 0.593 \\
\hline SEV (19) & 22.952 & 22.164 & 1.036 & 0.301 \\
\hline Year & 0.012 & 0.010 & 1.127 & 0.260 \\
\hline Year: FAR & -0.025 & 0.011 & -2.338 & 0.020 \\
\hline Year: WOO & 0.007 & 0.013 & 0.551 & 0.581 \\
\hline Year: SEV & -0.012 & 0.011 & -1.045 & 0.296 \\
\hline \multicolumn{5}{|c|}{ Additional tests } \\
\hline Null hypothesis & & & F Value & $p$ Value \\
\hline Year: FAR $=0$ & & & 18.505 & $<0.001$ \\
\hline Year: $\mathrm{WOO}=0$ & & & 5.513 & 0.019 \\
\hline Year: SEV = 0 & & & $<0.001$ & 0.977 \\
\hline Year: FAR = Year: WOO & & & 14.153 & $<0.001$ \\
\hline Year: FAR = Year: SEV & & & 7.572 & 0.006 \\
\hline Year: $\mathrm{WOO}=$ Year: SEV & & & 4.426 & 0.036 \\
\hline \multicolumn{5}{|c|}{ (D) Overall specialization index } \\
\hline \multicolumn{5}{|c|}{ Model output } \\
\hline Term & Estimate & SE & t Value & $p$ Value \\
\hline Intercept (19) & 4.256 & 9.046 & 0.471 & 0.638 \\
\hline INT (38) & 16.896 & 11.230 & 1.505 & 0.133 \\
\hline SPE (19) & 9.934 & 14.312 & 0.694 & 0.488 \\
\hline Year & -0.002 & 0.005 & -0.381 & 0.704 \\
\hline Year: INT & -0.008 & 0.006 & -1.511 & 0.131 \\
\hline Year: SPE & -0.005 & 0.007 & -0.691 & 0.490 \\
\hline \multicolumn{5}{|c|}{ Additional tests } \\
\hline Null hypothesis & & & F Value & $p$ Value \\
\hline Year: INT $=0$ & & & 9.411 & 0.002 \\
\hline Year: $\mathrm{SPE}=0$ & & & 1.446 & 0.230 \\
\hline Year: INT = Year: SPE & & & 0.300 & 0.584 \\
\hline
\end{tabular}




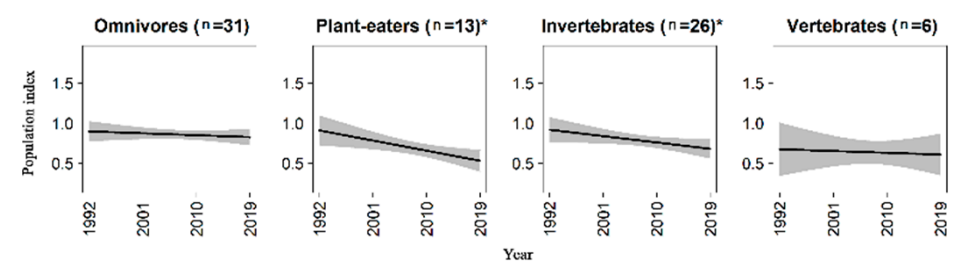

(A)
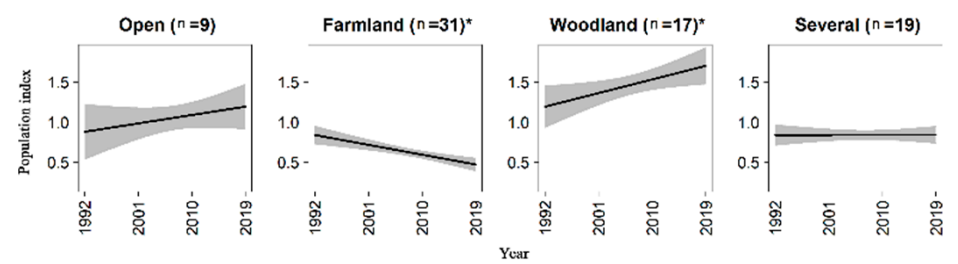

(C)
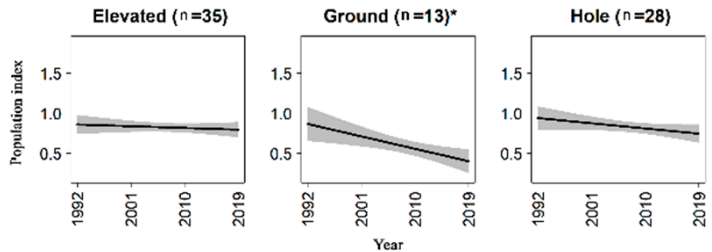

(B)
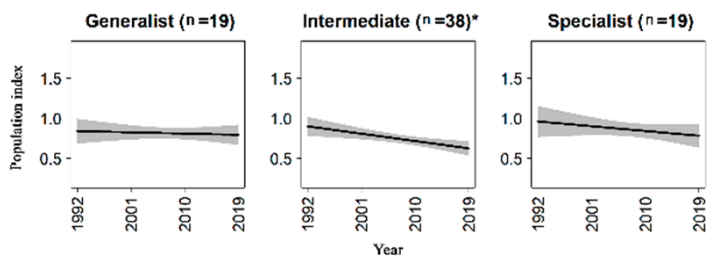

(D)

Figure 3. Population trends for bird species grouped according to ecological traits. (A) Diet; (B) nest type; (C) landscape type; (D) overall specialization index. Y-axis represents the population index of the species (see Section 2.4.2 for details). The number of species in each group is shown in parentheses. The asterisk indicates the significance of the trend in relation to non-zero slope.

\section{Discussion}

\subsection{Modelling Approach}

The use of adequate models to obtain reliable estimates of yearly population indices is fundamental to assess non-distorted population trends, whose evaluation is of extreme importance in wildlife conservation. In this context, our results showed that zero-inflated models should be used more than they actually are. Specifically, for $72 \%$ of the 76 species analyzed, zero-inflated GAMs resulted to be the most suitable models for predicting the yearly population indices. Unexpectedly, zero-inflated models are often overlooked in population trend analysis without an objective reason, but their use should be always tested in case of a high number of zero counts in the data. Overdispersion, which is often detected in count data deriving from multispecies surveys [58,97], can actually disappear or decrease if zero-inflated models are used (Table S4). The management of zero counts and the error structure for the response variable can strongly affect trend estimations [54], and it should not be carried out a priori but after a modelling selection procedure, which compares multiple candidate models.

\subsection{Species Population Trends}

The long-term trend analysis allowed obtaining an updated portrait of the conservation status of 76 common breeding birds in Lombardy. Although roughly three-quarters of them showed non-significant or increasing population trends, $24 \%$ of the studied species resulted on decline. The Eurasian Skylark (Alauda arvensis) showed a worrying trend: its population was reduced by $99.65 \%$ since 1992 , pointing out the extremely critical situation that the breeding populations of the species are experiencing in the Region. In a previous work, Bani et al. [58] found a reduction of $75 \%$ of the species regional population for the period from 1992 to 2009; after 2009, the population indices estimated by our analyses revealed a further decrease until 2015 (Figure S2), resulting in a stronger population decline, overall. However, at national and continental scale, the species exhibits a minor accentuated decrease [6,98]. In farmland, this species is very sensitive to the intensive agricultural management (with the use of pesticides, fertilizers, and excessive mowing) as well as to the decrease in crop diversity, both leading to unsuitable conditions for nesting [99-102]. Urbanization around arable land negatively affect the occurrence of the species [103], as well as the anthropic alterations in natural and semi-natural grassland in mountains areas [104]. In the Alpine area of Lombardy, the distribution of the Eurasian Skylark might be adversely affected by shrubs and forest expansion, as shown for other 
alpine species [12], but this pattern needs to be confirmed. Other five species (the African Stonechat Saxicola torquatus, the European Goldfinch Carduelis carduelis, the European Greenfinch Chloris chloris, the Eurasian Wryneck Jynx torquilla, and the Red-backed Shrike Lanius collurio) showed very strong negative trend, with population declines greater than $80 \%$. The African Stonechat experienced a sharp drop from 2006 (Figure S2). The decline resulted to be greater than reported at national scale in Italy, where a decrease of $68.5 \%$ was detected from 2000 to 2020 [98]. However, in the close Switzerland, a region environmentally and climatically similar to the Alpine area of Lombardy, the species appeared to be on increase [105], and in Europe it seemed to be stable [6]. These contrasting trends can derive from local factors (e.g., land management) that, acting at smaller spatial scale, can produce different effects in breeding areas [32]. For this species, land consolidation of arable fields, leading to the degradation of rural landscape structure, might act as ecological traps [106]. Trends for the European Goldfinch and the European Greenfinch showed a more drastic situation compared to the known status at national scale in the last twenty years [98]. The decline found by Bani et al. [58] in Lombardy seems to be continued over the last decade. However, in Europe these two finches did not decline over long period [6], or showed fluctuant dynamics, suggesting the existence of different drivers, such as seeds availability or epidemics, acting at local spatial scale [107]. In addition, both the Eurasian Wryneck and the Red-backed Shrike highlighted a more marked decline than that shown at national [98] and European [6] level. In the study area, the two species mainly inhabit semiopen environments, especially extensively managed farmland. Agricultural intensification, leading to loss of suitable foraging and nesting sites, as well as to a reduction of preys (mainly represented by insects) availability and detectability, may have played a key role in their decline [106,108-111]. We noted that other five species, the Barn Swallow (Hirundo rustica), the Western Yellow Wagtail (Motacilla flava), the Italian Sparrow, the Cetti's Warbler (Cettia cetti), and the Common Swift (Apus apus) experienced a significant decline ranging from $50 \%$ to $80 \%$, higher than the known situation at national scale $[98,112]$. The Barn Swallow, in our study area, showed a population decline of rough $50 \%$ during 1990 s and 2000s $[58,113,114]$, and the negative trend continued over the last decade. Causes can be linked to both changes in agricultural practices as well as cattle farming [115]. The Western Yellow Wagtail, which in Lombardy is associated to farmlands, may have suffered from the intensification and changes in agricultural practices, in particular from loss of late mown areas [116]. The Italian Sparrow, in agricultural breeding areas, could be negatively affected by the progressive shift of cereal cultivations from wheat to maize, other than changes in livestock farming practices [117]. In urban settlements, the food shortage (e.g., caused by a more efficient street cleaning and by a reduction in "weedy" areas), the reduction of suitable breeding sites, predation and competition had likely contributed to the species decline [117-119]. Both the Common Swift and the declining Common House Martin $(-45.55 \%)$ might have suffered from climatic factors acting in wintering and breeding grounds, but also along migration flyways $[120,121]$. Moreover, such species living in urban areas may suffer from different kinds of pollutants, as shown by Miniero et al. [122]. Less clear remain the drivers of the negative long-term trend of the Black Kite $(-54.96 \%)$, although some study highlighted the possible effect of electrocution and road casualty [123]. As for many other long-migrants species, we do not exclude that other factors might act in sub-Saharan wintering areas (see Section 4.2).

Among the species resulting on increase, the Great Cormorant exhibited a remarkable positive trend $(+12,060 \%)$ in the last fourteen years. This finding suggest the need of further and specific investigations in order to assess in detail the absolute dimension of the population, considering the impact that the species may have on herons [124], fish species [125] and economic activities as fisheries [126]. All tits (included the Long-tail Tit), resulted significantly on increase, except the Coal Tit (Periparus ater) that showed a positive but non-significant trend. These findings are largely in accordance with trends observed in Switzerland during the same period [105], while in Europe, during the last forty years, the Marsh Tit (Poecile palustris), the Willow Tit (Poecile montanus) and the 
European Crested Tit (Lophophanes cristatus) declined [6]. Also in Finland, these last two species declined due to intensive forestry [127], and the Willow Tit has been added in the National Red List [128]. We found a considerable increase of the population of the Northern Lapwing $(+1653 \%)$, a result quite in contrast with the negative trend observed in Europe [6], especially in arable land $[129,130]$, which is the type of habitat where the species mainly occurs in Lombardy. However, the first case of breeding of the Northern Lapwing in our study area is reported in 1960s, and from 1980s to the first decade of the twenty-first century the population has grown from a few hundreds of individuals to about two thousands of breeding pairs, probably supported by the water management of paddy fields, which represent the favorite breeding habitat for the species in Lombardy [131-133]. However, changes in agricultural practices for this kind of crops (in particular the delay of the flooding period) occurred in the last years, can negatively affect the reproductive success of the species, pointing out the importance to carry on specific demographic studies. Eurasian Magpie showed a strong increasing trend $(+754 \%)$. It resulted greater than the average increment at national scale [98], and in contrast with the European situation, where the species from the early 1990s exhibited a clear decrease [6]. In our region, where the urbanized areas increased during the last decades [55], the positive tendency of the Eurasian Magpie can be linked to its capacity of adapting to highly urbanized habitats. In this type of environment, the density of pairs can reach higher values than in non-urbanized habitats [134], maybe favored by high food availability. We have not evidence that the species increase is linked to a reduction of human persecution, as occurred in other areas (e.g., [135]). Song Thrush (Turdus philomelos), Common Wood Pigeon (Columba palumbus), Great Spotted Woodpecker (Dendrocopos major) and Short-toed Treecreeper showed strong positive trends, with regional population increased tenfold or more from the beginning of the time series. These findings confirmed the trends of the species in Europe, where from the early 1990s remarkable increments of the continental population were detected [6].

Population trends are one of the most important criteria for the conservation status assessment of species (e.g., [136]). Looking at the IUCN Italian Red List [137], we found that six of the 18 declining species are listed as "vulnerable", and one of them, the Eurasian Wryneck, as "engendered". The European Goldfinch and the European Greenfinch, which showed strong decreasing trends, are listed as "near threatened". Although our results are at regional scale, they can provide valuable information for updating local, national and continental Red Lists [39].

\subsection{Relation between Trends and Species Traits}

The trait-based approach proved to be very useful to infer on general drivers acting on wildlife populations, laying the foundations for more specific studies with the aim of identifying the proximal causes shaping bird population dynamic. Our analyses detected a general decline of migrant species, and a positive but non-significant trend for sedentary species. The decline of long-distance migrants has been extensively outlined for European breeding birds [42,138,139], with some exceptions (e.g., in Estonia [140]), and several explanations have been proposed (e.g., drought of Sahel areas, habitat loss and degradation, hunting, phenological mismatch [42,138,141-146]). Moreover, long-distance migrants were proved to decline faster than short-distance migrants in Europe [22,35,42], but we did not detect such a difference between the two groups, corroborating the idea that geographic variation in trends may occur [139].

The link between dispersal ratio, as surrogate of species' mobility, and the population trend did not highlighted a clear gradient for this trait. In a previous study, Crawford et al. [147] found that a lesser species' mobility was associated with a high rate of adult mortality. Although our results did not confirm this finding, and differences among the three groups did not emerge, a signal that long-term population dynamic can vary in relation to species' mobility was found for species with intermediate mobility. Further studies should focus on this life-history trait that is very poorly investigated in trend analysis. 
Species with high annual fecundity exhibited a clear negative trend. This finding was in accordance with results underlined by Jiguet et al. [89] for common breeding birds in France. Annual fecundity strongly influences variations in population size in short-lived species [148], and it is possible that individuals of such species will have too short a lifespan to learn and adapt to directional environmental changes. Moreover, density-dependent effects might also explain the observed pattern: high fecundity could lead to an increase in juvenile mortality, due to increased intraspecific competition [89,149].

Results for the incubation period highlighted that species with shorter incubation period decline, while those with longer incubation period showed a positive tendency, although it was not significant. On one hand, species with longer incubation period might be longer exposed to anthropogenic and ecological pressures during a critical stage of their life cycle. However, Liu et al. [150] highlighted that the incubation period is strongly and positively associated with nest concealment. Species that are able to hide their nests more efficiently, may be less exposed to predation risk, with positive effects on fitness in the long period. Additionally, shorter incubation periods are often found in species that face higher levels of nest predation $[151,152]$. However, predation risk may also be a habitat-dependent process [153]. Moreover, human activities can contribute to enhance this risk, as well as they can directly affect hatching success $[154,155]$. Further studies to investigate the effects of anthropogenic pressures on hatching success across different types of habitat can provide novel insights for planning ad hoc conservation strategies.

Analyses of ecological traits showed a decline of plant-eaters and species feeding on invertebrates. The decrease in insect populations has been largely recognized [156] and reported in several countries in Europe (e.g., [157,158]), as well as worldwide [159], but can only partially explain the general decrease of insectivore birds in Europe in the period 1990-2015 [37]. In our study, several species that eat invertebrates, such as the Northern Lapwing, the European Bee-eater (Merops apiaster), the Short-toed Treecreeper, the European Green Woodpecker (Picus viridis), the Spotted Flycatcher (Muscicapa striata), the Common Firecrest (Regulus ignicapilla) and the Melodius Warbler (Hippolais polyglotta) exhibited strong increasing trends $(>300 \%)$, highlighting that other component (e.g., habitat preferences) can interact with food habits in shaping population dynamic $[22,37,160]$. The decline of plant-eaters species (in our work mainly represented by species feeding on seeds) emerged in our study was in accordance with results in Europe [37]. In particular, plant-eaters linked with urban settlements and farmland, such as the Italian Sparrow, the European Goldfinch and the European Greenfinch, showed stronger negative trends. The use of herbicide and the disappearance of spring-sown cereals at the cost of winter-sown grains represent one of the major threats for the guild in farmland [161]. Additionally, the use of pesticides can have sub-lethal effects, such as impairing migratory ability in seed-eating species [162].

As regards to nest type, the general decrease of ground-nesters (largely farmland birds) can be associated with their higher predation risk $[45,150,163]$, which might also be linked to agricultural practices that reduce the concealment of nests by modifying the habitat structure [164].

Farmland species showed a strong decline (roughly equal to that of short-distance migrants, see Tables $2 \mathrm{~A}$ and $3 \mathrm{C}$ for comparison between regression coefficients). This finding is not surprising, since the decrease of farmland avifauna in Europe is well known [6,43], as well as documented in Italy in the last 20 years (with a stronger negative trend in Lombardy compared to the situation at national scale [98]). Causes of this decline are ascribed to agricultural intensification [161], which lead to simplification and homogenization of agricultural landscapes (land consolidation, loss of fallows), reduction of crop diversity and massive use of pesticides. Moreover, changes in agricultural management, such as increasing number of cuts, dense swards and reseeding with high-yielding grass crops, can lead to an increase of human disturbance and to a decrease of habitat suitability for many species. It is probably that all or a part of these processes have played, and still play, an important role in our study area, where intensive agriculture is widely spread. For example, 
in Lombardy the average harvest for maize and autumn-sown grains per hectare, thought to be a good indicator of agricultural intensity [165], was 9.3 ton/ha in the period 2006-2019, while the national average in the same period was 5.3 ton/ha [166]. However, 13 of the 31 farmland species showed significant positive trends, highlighting that species-specific drivers can occur and determine different demographic responses. For instance, some thermophilic farmland species, such as the European Bee-eater, showed strong positive trends, probably favored by temperature increasing [167-169]. Woodland birds, which appear stable or increasing in Europe $[22,43,170]$, confirmed this pattern in our study area. The increase in wood extension occurred in Lombardy over the last 40 years [55], both due to natural processes linked to the abandonment of mountain meadows and pastures and to human-made reforestations of bare grounds, may have increased the amount of suitable and available habitats for this group of species. This can have led to the establishment of new sub-populations and to the reduction of intraspecific and interspecific competition. Additionally, it is not to exclude that possible changes in forest management, resulting in an increase of forest quality, may have favored the positive trend of forest birds [171-174], but see [9]. Among woodland species, only two of them showed a negative trend: the Common Chiffchaff (Phylloscopus collybita), with a population reduction of $40.64 \%$, and the Goldcrest (Regulus regulus), with a decrease of $41.42 \%$. The Common Chiffchaff was also detected on decline by Bani et al. [58], but its trend is quite different compared to the observed trends in Europe [6], in Italy [112] from 2000 to 2014 and in other European countries (e.g., Germany [22], Switzerland [105], Sweden [175]). The species has many traits (long-distance migrants, intermediate dispersal ratio, ground-nesters, high annual fecundity, feeds on invertebrates) linked to negative trends, and it is probably that one or more of these species-specific characteristics are responsible of the observed negative trend in our study area. The Goldcrest experienced a general decline in Europe [6] as well as in Italy [112]. It is a cold adapted and forest specialist species, and the combination of these two factors may be the cause of the decline $[43,176]$. Species of natural-open habitat, which represent nine of the 11 species living in high mountain areas, did not show a significant trend. The finding is in accordance with that reported in some European mountains during the period 2002-2014 (i.e., Alps, Apennines), although in other mountain areas, such as Fennoscandia and Iberia, bird species declined significantly [177,178]. Indeed, mountain birds can differently respond to ecological drivers (e.g., climate change and land use changes), affecting local population trends and distribution [12,178].

Finally, we did not find a strong relationship between trends and the overall specialization index. This result did not fully support findings highlighted in previous studies. For example, Morelli et al. [40] underlined a negative relationship between the degree of specialization and population trends for 139 species in Europe, in particular in relation to nesting site specialization. Similarly, Kamp et al. [22] found a decline of specialist birds in relation to habitat breadth and diet breadth (note that categorization of these traits differs from the approach adopted in this study). It is clear that bird communities are moving to assemblages composed by more generalist species that are non-randomly replacing specialist ones [179], probably due to anthropogenic drivers such as habitat fragmentation that can more negatively affect specialists than generalists [25]. In our study, a signal that non-generalist species respond more adversely than generalist ones has been noted (Table 3D and Figure 3D).

\section{Conclusions}

The study provided an updating of long-term populations trends (1992-2019) for 76 common breeding birds in Lombardy using a statistical approach that allowed taking into account environmental bias, overdispersion and zero-inflation. Zero-inflated models largely resulted the best modelling choice in order to predict yearly population indices, and we encourage a broader application in population trend analysis than their actually employment. Overall, the bird conservation status in the region highlighted a favorable situation, with roughly three-quarters of the species showing positive or non-significant 
trends over the period. However, among the remnant species, 12 showed a decline greater than $50 \%$, and 6 experienced a worrying population reduction greater than $80 \%$, with the extreme drop of the Eurasian Skylark population (-99.65\%). Identifying the causes of the observed trends, especially when dramatically negative, is the foundation of any management plan and conservation action. In this context, a functional approach linking the species traits with the population trends allowed us to identify the most threatened groups. We found a decrease of migrant birds, species with high annual fecundity and short incubation period. Plant-eaters, species feeding on invertebrates and ground-nesters also decreased. Farmland birds showed a negative trend, while woodland species resulted on increase. A weaker signal was found in relation to the species' mobility and the degree of specialization. A trait-based approach should represent the starting point from which implement studies that test specific hypotheses aimed at identifying the direct causes responsible of the observed population dynamics. Further studies should focus on investigation of the relationship between long-term trends and species traits at large spatial scales, and on quantifying the effects of specific drivers across multiple species sharing similar functional characteristics.

Supplementary Materials: The following are available online at https://www.mdpi.com/article/ 10.3390/ani11123426/s1. Table S1. Number of point counts surveyed per year; Table S2: Monitoring programs from which bird data were derived; Table S3: Life history and ecological traits of the studied species; Figure S1: Cramer's V coefficients of the traits originally considered in the analysis; Table S4: Explained deviance (\%) and the Akaike Information Criterion (AIC) of the models performed for the 76 studied species; Table S5: Summary of models performed for each species; Figure S2: Plot of the population indices with confidence intervals and regression line for the 76 studied species.

Author Contributions: Conceptualization, P.T., L.B., V.O. and O.D.; methodology, P.T., L.B. and V.O.; software, P.T.; formal analysis, P.T.; investigation, L.B. and V.O.; data curation, L.B., V.O., L.K. and P.T.; writing-original draft preparation, P.T., L.B., V.O. and O.D.; writing-review and editing, L.B., V.O., O.D. and P.T.; supervision, L.B. All authors have read and agreed to the published version of the manuscript.

Funding: This research received no external funding.

Institutional Review Board Statement: Not applicable.

Data Availability Statement: Full dataset is available at the Department of Earth and Environmental Sciences, University of Milan-Bicocca, Piazza della Scienza 1, 20126, Milan, Italy, Building U1.

Acknowledgments: We thank the General Directorate for Agriculture of the Lombardy Region (D.G. Agricoltura della Regione Lombardia) and the Regional Agency for Agricultural and Forestry Development (ERSAF, Ente Regionale per lo Sviluppo Agricolo e Forestale) for co-funding bird data collection since 1992 through the monitoring project of breeding birds in Lombardy, also financed by the Research Fund of the University of Milano-Bicocca. We thank CINECA team for its support on Marconi HPC. We are very grateful to all the surveyors for their help with field data collection and particularly to Matteo Bonetti, Lucio Bordignon, Monica Carabella, Alberto Forelli, Lorenzo Fornasari, Sergio Mantovani and Renato Massa. We thank all bachelor's and master's students that contributed to data digitalization. We thank the editor Jukka Jokimäki and the three anonymous reviewers for their valuable comments and suggestions.

Conflicts of Interest: The authors declare no conflict of interest.

\section{Appendix A. Consideration on Detection Bias Issue in Population Trend Analysis}

The detection bias (i.e., the distortion due to factors that affect the species detection probability) represents an important issue in several studies e.g., [180-182]. However, at times, it is an overrated aspect, used unnecessarily at the expense of simplicity and cheapness. Detection bias can depend on species extrinsic or intrinsic factors. Extrinsic factors do not depend on the species behavior, and they include the surveyor ability, meteorological conditions (e.g., wind direction), environmental noises (e.g., vehicle traffic, waterfalls). Another important extrinsic factor to be considered is the variation of the 
detection probability according to the distance of the individual from the observer (each species has its specific detection probability function, that describes the relationship between the individual-observer distance and the probability to detect an individual in a specific habitat). Intrinsic factors rely on species behavior that directly affect the detection probability at the sampling site. They depend on the conspicuousness of a species, but can also be affected by extrinsic factors that can alter the species behavior. Accounting for all these potential sources of bias is crucial when the goal of a study is the assessment of the population density or an absolute magnitude, such as the population size, of a given species. To cope with such detection bias, multiple surveys of the same site along the same season or years are required [183].

When the goal of the study is the assessment of population trends (i.e., the relative variation of population size over time), the relative abundance is an adequate information, provided that it is estimated using data coming from a standardized sampling scheme. In this case, the sampling allows collecting representative information, comparable over time, since the survey technique does not change, and the same amount (or even proportion) of each habitat is taking into account in each year. This way, remaining the sampling design constant over time, the bias that would be produced by the differences in the species habitat-specific detection function, does not affect the trend estimation, since the relative abundance of a species is affected by the same detection bias over time.

However, when trend analysis relies on datasets deriving from many long-term monitoring programs, wherein the sampling design experienced some changes over time, or built using opportunistic data, the problem of obtaining a standardized-like dataset arise. The modelling approach applied in this research allows incorporating the detection bias arising from the species habitat-specific detection function, as well as the environmental bias among years (see Section 2.3 and [54]). Moreover, the effect of detection bias arising from the above-mentioned extrinsic factors, out of the detection probability function, could be difficult to manage (but see $\mathrm{N}$-mixture models $[183,184]$ ), but it can be considered negligible when statistical inferences are applied on large datasets [25].

Finally, in the case of monitoring programs performed at large spatial scale, the use of the unlimited distance technique [56], prevent from the introduction of further source of bias. Indeed, when several surveyors are involved in data collection, a rough estimate by eye of detection distance on the field (e.g., fixed radius) could differ among observers, leading to a bias that would be difficult to deal with. Therefore, in our opinion, it would be helpful to promote studies aimed at estimating the species habitat-specific detection function, along with the collection of data without distance limits. This is a crucial point to obtain population density or size.

\section{References}

1. Donald, P.F.; Sanderson, F.J.; Burfield, I.J.; Bierman, S.M.; Gregory, R.D.; Waliczky, Z. International conservation policy delivers benefits for birds in Europe. Science 2007, 317, 810-813. [CrossRef]

2. Stanton, R.L.; Morrissey, C.A.; Clark, R.G. Analysis of trends and agricultural drivers of farmland bird declines in North America: A review. Agric. Ecosyst. Environ. 2018, 254, 244-254. [CrossRef]

3. Rosenberg, K.V.; Dokter, A.M.; Blancher, P.J.; Sauer, J.R.; Smith, A.C.; Smith, P.A.; Stanton, J.C.; Panjabi, A.; Helft, L.; Parr, M.; et al. Decline of the North American avifauna. Science 2019, 366, 120-124. [CrossRef]

4. Di Marco, M.; Butt, N.; Possingham, H.P.; Kearney, S.; Watson, J.E.M. Changing trends and persisting biases in three decades of conservation science. Glob. Ecol. Conserv. 2017, 10, 32-42. [CrossRef]

5. Callaghan, C.T.; Nakagawa, S.; Cornwell, W.K. Global abundance estimates for 9700 bird species. Proc. Natl. Acad. Sci. USA 2021, 118, e2023170118. [CrossRef] [PubMed]

6. Pan European Common Bird Monitoring Scheme. Available online: https://pecbms.info/ (accessed on 21 September 2021).

7. Sauer, J.R.; Pardieck, K.L.; Ziolkowski, D.J., Jr.; Smith, A.C.; Hudson, M.-A.R.; Rodriguez, V.; Berlanga, H.; Niven, D.K.; Link, W.A. The first 50 years of the North American breeding bird survey. Condor Ornithol. Appl. 2017, 119, 576-593. [CrossRef]

8. Fraixedas, S.; Lehikoinen, A.; Lindén, A. Impacts of climate and land-use change on wintering bird populations in Finland. J. Avian Biol. 2015, 46, 63-72. [CrossRef]

9. Fraixedas, S.; Lindén, A.; Lehikoinen, A. Population trends of common breeding forest birds in southern Finland are consistent with trends in forest management and climate change. Ornis Fenn. 2015, 92, 187-203. 
10. Harris, S.J.; Massimino, D.; Balmer, D.E.; Eaton, M.A.; Noble, D.G.; Pearce-Higgins, J.W.; Woodcock, P.; Gillings, S. The Breeding Bird Survey 2019. BTO Research Report 726. British Trust for Ornithology; Thetford: Norfolk, UK, 2019.

11. Massimino, D.; Orioli, V.; Massa, R.; Bani, L. Population trend assessment on a large spatial scale: Integrating data collected with heterogeneous sampling schemes by means of habitat modelling. Ethol. Ecol. Evol. 2008, 20, 141-153. [CrossRef]

12. Bani, L.; Luppi, M.; Rocchia, E.; Dondina, O.; Orioli, V. Winners and losers: How the elevational range of breeding birds on Alps has varied over the past four decades due to climate and habitat changes. Ecol. Evol. 2019, 9, 1289-1305. [CrossRef]

13. Sibilia, A.; Orioli, V.; Trasforini, S.; Puzzi, C.M.; Bani, L. The distribution and richness of the Italian riverine fish provided by the BioFresh database. Folia Zool. 2019, 68, 225-234. [CrossRef]

14. Bani, L.; Orioli, V.; Trasforini, S.; Puzzi, C.M.; Sibilia, A.; Dondina, O.; Tirozzi, P. The spread of exotic fish species in Italian rivers and their effect on native fish fauna since 1990. Biodiversity 2020, 1-9. [CrossRef]

15. Stefani, F.; Schiavon, A.; Tirozzi, P.; Gomarasca, S.; Marziali, L. Functional response of fish communities in a multistressed freshwater world. Sci. Total Environ. 2020, 740, 139902. [CrossRef] [PubMed]

16. Burger, J.; Gochfeld, M. Marine Birds as Sentinels of Environmental Pollution. Ecohealth 2004, 1, 263-274. [CrossRef]

17. Dondina, O.; Orioli, V.; Massimino, D.; Pinoli, G.; Bani, L. A method to evaluate the combined effect of tree species composition and woodland structure on indicator birds. Ecol. Indic. 2015, 55, 44-51. [CrossRef]

18. Natsukawa, H. Raptor breeding sites indicate high taxonomic and functional diversities of wintering birds in urban ecosystems. Urban For. Urban Green. 2021, 60, 127066. [CrossRef]

19. Oettel, J.; Lapin, K. Linking forest management and biodiversity indicators to strengthen sustainable forest management in Europe. Ecol. Indic. 2021, 122, 107275. [CrossRef]

20. Santangeli, A.; Girardello, M. The representation potential of raptors for globally important nature conservation areas. Ecol. Indic. 2021, 124, 107434. [CrossRef]

21. Krebs, C.J. The experimental paradigm and long-term population studies. IBIS 1991, 133, 3-8. [CrossRef]

22. Kamp, J.; Frank, C.; Trautmann, S.; Busch, M.; Dröschmeister, R.; Flade, M.; Gerlach, B.; Karthäuser, J.; Kunz, F.; Mitschke, A.; et al. Population trends of common breeding birds in Germany 1990-2018. J. Ornithol. 2021, 162, 1-15. [CrossRef]

23. Villéger, S.; Mason, N.W.H.; Mouillot, D. New multidimensional functional diversity indices for a multifaceted framework in functional ecology. Ecology 2008, 89, 2290-2301. [CrossRef] [PubMed]

24. Gagic, V.; Bartomeus, I.; Jonsson, T.; Taylor, A.; Winqvist, C.; Fischer, C.; Slade, E.M.; Steffan-Dewenter, I.; Emmerson, M.; Potts, S.G.; et al. Functional identity and diversity of animals predict ecosystem functioning better than species-based indices. Proc. R. Soc. B Biol. Sci. 2015, 282, 20142620. [CrossRef]

25. Dondina, O.; Orioli, V.; D'Occhio, P.; Luppi, M.; Bani, L. How does forest species specialization affect the application of the island biogeography theory in fragmented landscapes? J. Biogeogr. 2017, 44, 1041-1052. [CrossRef]

26. Jacoboski, L.I.; Hartz, S.M. Using functional diversity and taxonomic diversity to assess effects of afforestation of grassland on bird communities. Perspect. Ecol. Conserv. 2020, 18, 103-108. [CrossRef]

27. Betancurt-Grisales, J.F.; Vargas-Daza, A.M.; Castaño-Villa, G.J.; Ospina-Bautista, F. Bird functional diversity in restored and secondary forests of the Colombian Andes. Restor. Ecol. 2021, 29, e13315. [CrossRef]

28. Leveau, L.M. Consistency in bird community assembly over medium-term along rural-urban gradients in Argentina. Ecol. Process 2021, 10, 34. [CrossRef]

29. Jokimäki, J.; Suhonen, J.; Jokimäki-Kaisanlahti, M.-L.; Carbó-Ramírez, P. Effects of urbanization on breeding birds in European towns: Impacts of species traits. Urban Ecosyst. 2016, 19, 1565-1577. [CrossRef]

30. Pocock, M.J.O. Can traits predict species' vulnerability? A test with farmland passerines in two continents. Proc. R. Soc. B Biol. Sci. 2011, 278, 1532-1538. [CrossRef]

31. Sullivan, M.J.P.; Newson, S.E.; Pearce-Higgins, J.W. Using habitat-specific population trends to evaluate the consistency of the effect of species traits on bird population change. Biol. Conserv. 2015, 192, 343-352. [CrossRef]

32. Rocchia, E.; Luppi, M.; Dondina, O.; Orioli, V.; Bani, L. Can the effect of species ecological traits on birds' altitudinal changes differ between geographic areas? Acta Oecologica 2018, 92, 26-34. [CrossRef]

33. Hanzelka, J.; Horká, P.; Reif, J. Spatial gradients in country-level population trends of European birds. Divers. Distrib. 2019, 25, 1527-1536. [CrossRef]

34. Vergara-Tabares, D.L.; Cordier, J.M.; Landi, M.A.; Olah, G.; Nori, J. Global trends of habitat destruction and consequences for parrot conservation. Glob. Chang. Biol. 2020, 26, 4251-4262. [CrossRef] [PubMed]

35. Flousek, J.; Telenský, T.; Hanzelka, J.; Reif, J. Population Trends of Central European Montane Birds Provide Evidence for Adverse Impacts of Climate Change on High-Altitude Species. PLoS ONE 2015, 10, e0139465. [CrossRef] [PubMed]

36. Soykan, C.U.; Sauer, J.; Schuetz, J.G.; LeBaron, G.S.; Dale, K.; Langham, G.M. Population trends for North American winter birds based on hierarchical models. Ecosphere 2016, 7, e01351. [CrossRef]

37. Bowler, D.E.; Heldbjerg, H.; Fox, A.D.; de Jong, M.; Böhning-Gaese, K. Long-term declines of European insectivorous bird populations and potential causes. Conserv. Biol. 2019, 33, 1120-1130. [CrossRef]

38. Garcia-R, J.C.; Di Marco, M. Drivers and trends in the extinction risk of New Zealand's endemic birds. Biol. Conserv. 2020, 249, 108730. [CrossRef]

39. Vavylis, D.; Bounas, A.; Karris, G.; Triantis, K.A. The state of breeding birds in Greece: Trends, threats, and implications for conservation. Bird Conserv. Int. 2020, 1-15. [CrossRef] 
40. Morelli, F.; Benedetti, Y.; Callaghan, C.T. Ecological specialization and population trends in European breeding birds. Glob. Ecol. Conserv. 2020, 22, e00996. [CrossRef]

41. Dumandan, P.K.T.; Bildstein, K.L.; Goodrich, L.J.; Zaiats, A.; Caughlin, T.T.; Katzner, T.E. Shared functional traits explain synchronous changes in long-term count trends of migratory raptors. Glob. Ecol. Biogeogr. 2021, 30, 640-650. [CrossRef]

42. Sanderson, F.J.; Donald, P.F.; Pain, D.J.; Burfield, I.J.; van Bommel, F.P.J. Long-term population declines in Afro-Palearctic migrant birds. Biol. Conserv. 2006, 131, 93-105. [CrossRef]

43. Gregory, R.D.; Skorpilova, J.; Vorisek, P.; Butler, S. An analysis of trends, uncertainty and species selection shows contrasting trends of widespread forest and farmland birds in Europe. Ecol. Indic. 2019, 103, 676-687. [CrossRef]

44. Liordos, V.; Jokimäki, J.; Kaisanlahti-Jokimäki, M.-L.; Valsamidis, E.; Kontsiotis, V.J. Niche analysis and conservation of bird species using urban core areas. Sustainability 2021, 13, 6327. [CrossRef]

45. Roos, S.; Smart, J.; Gibbons, D.W.; Wilson, J.D. A review of predation as a limiting factor for bird populations in mesopredator-rich landscapes: A case study of the UK. Biol. Rev. 2018, 93, 1915-1937. [CrossRef] [PubMed]

46. Chamberlain, D.E.; Fuller, R.J.; Bunce, R.G.H.; Duckworth, J.C.; Shrubb, M. Changes in the abundance of farmland birds in relation to the timing of agricultural intensification in England and Wales. J. Appl. Ecol. 2000, 37, 771-788. [CrossRef]

47. Isaksson, D.; Wallander, J.; Larsson, M. Managing predation on ground-nesting birds: The effectiveness of nest exclosures. Biol. Conserv. 2007, 136, 136-142. [CrossRef]

48. Macdonald, M.A.; Bolton, M. Predation on wader nests in Europe. IBIS 2008, 150, 54-73. [CrossRef]

49. Kaasiku, T.; Rannap, R.; Kaart, T. Managing coastal grasslands for an endangered wader species can give positive results only when expanding the area of open landscape. J. Nat. Conserv. 2019, 48, 12-19. [CrossRef]

50. Warren, P.; Land, C.; Hesford, N.; Baines, D. Conserving Black Grouse Lyrurus tetrix in southern Scotland: Evidence for the need to retain large contiguous moorland habitat within a forest-moorland landscape. Bird Study 2019, 66, 494-502. [CrossRef]

51. Jahren, T.; Storaas, T.; Willebrand, T.; Fossland Moa, P.; Hagen, B.-R. Declining reproductive output in capercaillie and black grouse-16 countries and 80 years. Anim. Biol. 2016, 66, 363-400. [CrossRef]

52. Fornasari, L.; de Carli, E.; Brambilla, S.; Buvoli, L.; Maritan, E.; Mingozzi, T. Distribuzione dell'avifauna nidificante in Italia: Primo bollettino del progetto di monitoraggio MITO2000. Avocetta 2002, 26, 59-115.

53. Fornasari, L.; de Carli, E.; Buvoli, L.; Mingozzi, T.; Pedrini, P.; La Gioia, G.; Ceccarelli, P.; Tellini Florenzano, G.; Velatta, F.; Caliendo, M.F.; et al. Secondo bollettino del progetto MITO2000: Valutazioni metodologiche per il calcolo delle variazioni interannuali. Avocetta 2004, 28, 59-76.

54. Tirozzi, P.; Orioli, V.; Dondina, O.; Kataoka, L.; Bani, L. Population trends from count data: Handling environmental bias, overdispersion and zero-inflation. Ecol. Inform. 2021. under review, 2nd round.

55. ERSAF. Uso del suolo in Regione Lombardia. I dati DUSAF, Destinazione d'Uso dei Suoli Agricoli e Forestali. 2018. Available online: https: / / www.geoportale.regione.lombardia.it/ (accessed on 21 September 2021).

56. Blondel, J.; Ferry, C.; Frochot, B. Point counts with unlimited distance. Stud Avian Biol 1981, 6, 414-420.

57. Fornasari, L.; Bani, L.; De Carli, E.; Massa, R. Optimum design in monitoring common birds and their habitat. Gibier Faune Sauvag. 1998, 15, 309-322.

58. Bani, L.; Massimino, D.; Orioli, V.; Bottoni, L.; Massa, R. Assessment of population trends of common breeding birds in Lombardy, Northern Italy, 1992-2007. Ethol. Ecol. Evol. 2009, 21, 27-44. [CrossRef]

59. Blondel, J.; Ferry, C.; Frochot, B. La méthode des indices ponctuels d'abondance (IPA) ou des relevés d'avifaune par "stations d'écoute". Alauda 1970, 38, 55-71.

60. Bibby, C.J.; Burgess, N.D.; Hill, D.A.; Hillis, D.M.; Mustoe, S. Bird Census Techniques; Academic Press: London, UK, 2000; ISBN 0120958317.

61. Kéry, M.; Dorazio, R.M.; Soldaat, L.; Van Strien, A.; Zuiderwijk, A.; Royle, J.A. Trend estimation in populations with imperfect detection. J. Appl. Ecol. 2009, 46, 1163-1172. [CrossRef]

62. Bani, L. Problemi e Metodi per un Conteggio a Lungo Termine Degli Uccelli Nidificanti in Lombardia. Master's Thesis, University of Milan, Milan, Italy, 1995.

63. Phillips, S.J.; Dudík, M.; Elith, J.; Graham, C.H.; Lehmann, A.; Leathwick, J.; Ferrier, S. Sample selection bias and presence-only distribution models: Implications for background and pseudo-absence data. Ecol. Appl. 2009, 19, 181-197. [CrossRef]

64. Kramer-Schadt, S.; Niedballa, J.; Pilgrim, J.D.; Schröder, B.; Lindenborn, J.; Reinfelder, V.; Stillfried, M.; Heckmann, I.; Scharf, A.K.; Augeri, D.M. The importance of correcting for sampling bias in MaxEnt species distribution models. Divers. Distrib. 2013, 19, 1366-1379. [CrossRef]

65. Zuur, A.; Ieno, E.N.; Walker, N.; Saveliev, A.A.; Smith, G.M. Mixed Effects Models and Extensions in Ecology with R; Springer Science \& Business Media: Berlin/Heidelberg, Germany, 2009.

66. Martin, T.G.; Wintle, B.A.; Rhodes, J.R.; Kuhnert, P.M.; Field, S.A.; Low-Choy, S.J.; Tyre, A.J.; Possingham, H.P. Zero tolerance ecology: Improving ecological inference by modelling the source of zero observations. Ecol. Lett. 2005, 8, 1235-1246. [CrossRef]

67. Denes, F.V.; Silveira, L.F.; Beissinger, S.R. Estimating abundance of unmarked animal populations: Accounting for imperfect detection and other sources of zero inflation. Methods Ecol. Evol. 2015, 6, 543-556. [CrossRef]

68. Blasco-Moreno, A.; Pérez-Casany, M.; Puig, P.; Morante, M.; Castells, E. What does a zero mean? Understanding false, random and structural zeros in ecology. Methods Ecol. Evol. 2019, 10, 949-959. [CrossRef]

69. Hastie, T.J.; Tibshirani, R.J. Generalized Additive Models. Stat. Sci. 1986, 1, 297-310. [CrossRef] 
70. Hastie, T.J.; Tibshirani, R.J. Generalized Additive Models; CRC Press: Boca Raton, FL, USA, 1990; Volume 43, p. 352 , ISBN 0412343908.

71. Wood, S.N. Generalized Additive Models: An Introduction with R; CRC Press: Boca Raton, FL, USA, 2017; p. 476, ISBN 1498728340.

72. McCullagh, P.; Nelder, J.A. Generalized Linear Models, 2nd ed.; CRC Press: Boca Raton, FL, USA, 1982.

73. Dormann, F.C.; McPherson, M.J.; Araújo, B.M.; Bivand, R.; Bolliger, J.; Carl, G.; Davies, G.R.; Hirzel, A.; Jetz, W.; Daniel Kissling, W. Methods to account for spatial autocorrelation in the analysis of species distributional data: A review. Ecography 2007, 30, 609-628. [CrossRef]

74. Burnham, K.P.; Anderson, D.R. Model Selection and Multimodel Inference: A Practical Information-Theoretic Approach, 2nd ed.; Springer Science \& Business Media: Berlin/Heidelberg, Germany, 2002; p. 488, ISBN 978-0-387-22456-5.

75. Nelson, W.A. Statistical Methods. In Encyclopedia of Ecology, 1st ed.; Jørgensen, S.E., Fath, B., Eds.; Elsevier: Oxford, UK, 2008; pp. 3350-3362, ISBN 9780080454054.

76. Davison, A.C.; Hinkley, D.V. Bootstrap Methods and Their Application; Cambridge University Press: New York, NY, USA, 2006; pp. 191-251.

77. Byrkjedal, I.; Kyllingstad, K.; Efteland, S.; Grøsfjell, S. Population trends of northern lapwing, Eurasian curlew and Eurasian oystercatcher over 15 years in a southwest Norwegian farmland. Ornis Nor. 2012, 35, 16-22. [CrossRef]

78. Lockerbie, E.M.; Shannon, L.J.; Jarre, A. The use of ecological, fishing and environmental indicators in support of decision making in southern Benguela fisheries. Ecol. Indic. 2016, 69, 473-487. [CrossRef]

79. R Core Development Team. R: A Language and Environment for Statistical Computing. Vienna, Austria: R Foundation for Statistical Computing. 2020. Available online: https:/ / www.R-project.org/ (accessed on 21 September 2021).

80. Wood, S. Mixed GAM Computation Vehicle with Automatic Smoothness Estimation. R Package Version 1.8-38. 2021. Available online: https:/ / cran.r-project.org/web/packages/mgcv/mgcv.pdf (accessed on 7 October 2021).

81. Wotherspoon, S.; Burch, P. EM Implementation of Zero-Inflated GAMs. R Package Version 0.1.1. 2016. Available online: https://github.com/AustralianAntarcticDataCentre/zigam/ (accessed on 21 September 2021).

82. CINECA. 2020. Available online: https://www.hpc.cineca.it/hardware/marconi (accessed on 21 September 2021).

83. Cramér, H. Mathematical Methods of Statistics, 1st ed.; Princeton University Press: Princeton, NJ, USA, 1946 ; p. 575.

84. Meyer, D.; Zeileis, A.; Hornik, K. Visualizing Categorical Data, R Package Version 1.4-8. 2020. Available online: https://cran.rproject.org/web/packages/vcd/citation.html (accessed on 21 September 2021).

85. Cohen, J. Statistical Power Analysis for the Behavioral Sciences, 2nd ed.; Taylor \& Francis Inc.: Hillsdale, NJ, USA, $1988 ;$ p. 400.

86. Gejdoš, M.; Suchomel, J.; Danihelová, Z. Analysis of qualitative features of beech and oak trunks as a determinant of the quality assessment. Forests 2021, 12, 15. [CrossRef]

87. VIGORITA, V.-C.L. La Fauna Selvatica in Lombardia. Rapporto 2008 su Distribuzione, Abbondanza e Stato di Conservazione di Uccelli e Mammiferi; Regione Lombardia: Milan, Italy, 2008.

88. Li, C.; Zhao, B.; Wang, Y. Nestedness of waterbird assemblages in the subsidence wetlands recently created by underground coal mining. Curr. Zool. 2018, 65, 155-163. [CrossRef]

89. Jiguet, F.; Gadot, A.-S.; Julliard, R.; Newson, S.E.; Couvet, D. Climate envelope, life history traits and the resilience of birds facing global change. Glob. Chang. Biol. 2007, 13, 1672-1684. [CrossRef]

90. Storchová, L.; Hořák, D. Life-history characteristics of European birds. Glob. Ecol. Biogeogr. 2018, 27, 400-406. [CrossRef]

91. Wilman, H.; Belmaker, J.; Simpson, J.; de la Rosa, C.; Rivadeneira, M.M.; Jetz, W. EltonTraits 1.0: Species-level foraging attributes of the world's birds and mammals. Ecology 2014, 95, 2027. [CrossRef]

92. Pearman, P.B.; Lavergne, S.; Roquet, C.; Wüest, R.; Zimmermann, N.E.; Thuiller, W. Phylogenetic patterns of climatic, habitat and trophic niches in a European avian assemblage. Glob. Ecol. Biogeogr. 2014, 23, 414-424. [CrossRef] [PubMed]

93. CORINE Land Cover. Available online: https://land.copernicus.eu/pan-european/corine-land-cover (accessed on 21 September 2021).

94. Julliard, R.; Clavel, J.; Devictor, V.; Jiguet, F.; Couvet, D. Spatial segregation of specialists and generalists in bird communities. Ecol. Lett. 2006, 9, 1237-1244. [CrossRef]

95. Fox, J.; Wieisberg, S.; Price, B. Companion to Applied Regression, R Package Version 3.0-8. 2020. Available online: https: / / cran.r-project.org/web/packages/car/index.html (accessed on 21 September 2021).

96. Gill, F.; Donsker, D.; Rasmussen, P. IOC World Bird List (v11.2). 2021. Available online: https://doi.org/10.14344/IOC.ML.11.2 (accessed on 21 September 2021).

97. Wagh, Y.S.; Kamalja, K.K. Zero-inflated models and estimation in zero-inflated Poisson distribution. Commun. Stat. Comput. 2018, 47, 2248-2265. [CrossRef]

98. Rete Rurale Nazionale \& Lipu. Common Breeding Farmland Birds in Italy. Update of Population Trends and Farmland Bird Indicator for National Rural Network 2000-2020. 2020. Available online: https:/ /www.reterurale.it/flex/cm/pages/ServeBLOB.php/L/IT/ IDPagina/22311 (accessed on 21 September 2021).

99. Chamberlain, D.E.; Siriwardena, G.M. The effects of agricultural intensification on Skylarks (Alauda arvensis): Evidence from monitoring studies in Great Britain. Environ. Rev. 2000, 8, 95-113. [CrossRef]

100. Chamberlain, D.E.; Vickery, J.A.; Gough, S. Spatial and temporal distribution of breeding Skylarks Alauda arvensis in relation to crop type in periods of population increase and decrease. Ardea 2000, 88, 61-73.

101. Eraud, C.; Marie Boutin, J. Density and productivity of breeding Skylarks Alauda arvensis in relation to crop type on agricultural lands in western France. Bird Study 2002, 49, 287-296. [CrossRef] 
102. Koleček, J.; Reif, J.; Weidinger, K. The abundance of a farmland specialist bird, the skylark, in three European regions with contrasting agricultural management. Agric. Ecosyst. Environ. 2015, 212, 30-37. [CrossRef]

103. Loretto, M.-C.; Schöll, E.M.; Hille, S. Occurrence of Eurasian Skylark Alauda arvensis territories in relation to urban area and heterogeneous farmland. Bird Study 2019, 66, 273-278. [CrossRef]

104. Brambilla, M.; Gustin, M.; Cento, M.; Ilahiane, L.; Celada, C. Habitat, climate, topography and management differently affect occurrence in declining avian species: Implications for conservation in changing environments. Sci. Total Environ. 2020, 742, 140663. [CrossRef]

105. Knaus, P.T.; Sattler, H.; Schmid, N.; Strebel, N.; Volet, B. The State of Birds in Switzerland: Report 2021; Swiss Ornithological Institute: Sempach, Switzerland, 2021.

106. Denac, K.; Kmecl, P. Land consolidation negatively affects farmland bird diversity and conservation value. J. Nat. Conserv. 2021, 59, 125934. [CrossRef]

107. Lehikoinen, A.; Lehikoinen, E.; Valkama, J.; Väisänen, R.A.; Isomursu, M. Impacts of trichomonosis epidemics on Greenfinch Chloris chloris and Chaffinch Fringilla coelebs populations in Finland. IBIS 2013, 155, 357-366. [CrossRef]

108. Coudrain, V.; Arlettaz, R.; Schaub, M. Food or nesting place? Identifying factors limiting Wryneck populations. J. Ornithol. 2010, 151, 867-880. [CrossRef]

109. Weisshaupt, N.; Arlettaz, R.; Reichlin, T.S.; Tagmann-Ioset, A.; Schaub, M. Habitat selection by foraging Wrynecks Jynx torquilla during the breeding season: Identifying the optimal habitat profile. Bird Study 2011, 58, 111-119. [CrossRef]

110. Le Roux, D.S.; Ikin, K.; Lindenmayer, D.B.; Bistricer, G.; Manning, A.D.; Gibbons, P. Enriching small trees with artificial nest boxes cannot mimic the value of large trees for hollow-nesting birds. Restor. Ecol. 2016, 24, 252-258. [CrossRef]

111. Bakx, T.R.M.; Lindström, Å.; Ram, D.; Pettersson, L.B.; Smith, H.G.; van Loon, E.E.; Caplat, P. Farmland birds occupying forest clear-cuts respond to both local and landscape features. For. Ecol. Manag. 2020, 478. [CrossRef]

112. MITO2000. Available online: https:// mito2000.it/andamenti/specie-target/ (accessed on 1 October 2021).

113. Ambrosini, R.; Rubolini, D.; Trovò, P.; Liberini, G.; Bandini, M.; Romano, A.; Sicurella, B.; Scandolara, C.; Romano, M.; Saino, N. Maintenance of livestock farming may buffer population decline of the Barn Swallow Hirundo rustica. Bird Conserv. Int. 2012, 22, 411-428. [CrossRef]

114. Sicurella, B.; Caprioli, M.; Romano, A.; Romano, M.; Rubolini, D.; Saino, N.; Ambrosini, R. Hayfields enhance colony size of the Barn Swallow Hirundo rustica in northern Italy. Bird Conserv. Int. 2014, 24, 17-31. [CrossRef]

115. Ambrosini, R.; Bani, L.; Massimino, D.; Fornasari, L.; Saino, N. Large-scale spatial distribution of breeding Barn Swallows Hirundo rustica in relation to cattle farming. Bird Study 2011, 58, 495-505. [CrossRef]

116. Broyer, J.; Sukhanova, O.; Mischenko, A. How to sustain meadow passerine populations in Europe through alternative mowing management. Agric. Ecosyst. Environ. 2016, 215, 133-139. [CrossRef]

117. Brlík, V.; Šilarová, E.; Škorpilová, J.; Alonso, H.; Anton, M.; Aunins, A.; Benkö, Z.; Biver, G.; Busch, M.; Chodkiewicz, T.; et al. Long-term and large-scale multispecies dataset tracking population changes of common European breeding birds. Sci. Data 2021, 8, 21. [CrossRef]

118. Wilkinson, N. Factors influencing the small-scale distribution of House Sparrows Passer domesticus in a suburban environment. Bird Study 2006, 53, 39-46. [CrossRef]

119. Dinetti, M. The Sparrows Passer spp.: From "pest species" to species of conservation concern. Avocetta 2008, 32, 61-68.

120. Rajchard, J.; Procházka, J.; Kindlmann, P. Long-term decline in Common Swift Apus apus annual breeding success may be related to weather conditions. Ornis Fenn. 2006, 83, 66-72.

121. Ambrosini, R.; Orioli, V.; Massimino, D.; Bani, L. Identification of putative wintering areas and ecological determinants of population dynamics of Common House-Martin (Delichon urbicum) and Common Swift (Apus apus) breeding in northern Italy. Avian Conserv. Ecol. 2011, 6. [CrossRef]

122. Miniero, R.; Carere, C.; De Felip, E.; Iacovella, N.; Rodriguez, F.; Alleva, E.; Di Domenico, A. The use of common swift (Apus apus), an aerial feeder bird, as a bioindicator of persistent organic microcontaminants. Ann. Dell'istituto Super. Sanità 2008, 44, 187-194.

123. De Pascalis, F.; Panuccio, M.; Bacaro, G.; Monti, F. Shift in proximate causes of mortality for six large migratory raptors over a century. Biol. Conserv. 2020, 251, 108973. [CrossRef]

124. Giammarino, M.; Quatto, P.; Renna, M. Impacts of Great Cormorant and Cattle Egret Nesting on Other Waterbirds in a Shared Breeding Site in Piedmont (NW Italy). Acta Ornithol. 2021, 56, 39-50. [CrossRef]

125. Delmastro, G.B.; Boano, G.; Conte, P.L.; Fenoglio, S. Great cormorant predation on Cisalpine pike: A conservation conflict. Eur. J. Wildl. Res. 2015, 61, 743-748. [CrossRef]

126. Veneranta, L.; Heikinheimo, O.; Marjomäki, T.J. Cormorant (Phalacrocorax carbo) predation on a coastal perch (Perca fluviatilis) population: Estimated effects based on PIT tag mark-recapture experiment. ICES J. Mar. Sci. 2020, 77, 2611-2622. [CrossRef]

127. Laaksonen, T.; Lehikoinen, A. Population trends in boreal birds: Continuing declines in agricultural, northern, and long-distance migrant species. Biol. Conserv. 2013, 168, 99-107. [CrossRef]

128. Tiainen, J.; Mikkola-Roos, M.; Below, A.; Jukarainen, A.; Lehikoinen, A.; Lehtiniemi, T.; Pessa, J.; Rajasärkkä, A.; Rintala, J.; Sirkiä, P.; et al. Suomen Lintujen Uhanalaisuus 2015-The 2015 Red List of Finnish Bird Species; Ympäristöministeriö \& Suomen Ympäristökeskus: Helsinki, Finland, 2016; p. 49.

129. Thorup, O. Population sizes and trends of breeding meadow birds in Denmark. Wader Study 2018, 125, 175-189. [CrossRef] 
130. Lislevand, T.; Byrkjedal, I.; Heggøy, O.; Kålås, J.A. Population status, trends and conservation of meadow-breeding waders in Norway. Wader Study 2021, 128, 6-21. [CrossRef]

131. Brichetti, P.; Fracasso, C. Ornitologia Italiana Volume II, Tetraonida-Scolopacidae; Alberto Perdisa Editore: Bologna, Italy, $2004 ;$ p. 165.

132. Longoni, V.; Serrano, S.; Vigorita, V.; Cucé, L.; Fasola, M. Ecologia e Popolazioni Della Pavoncella Vanellus Vanellus, Specie D'interesse Venatorio, in Regione Lombardia; Regione Lombardia: Milan, Italy, 2011. Available online: https:/ /www.regione.lombardia.it/wps/ wcm/connect/6c04d1f4-c914-4e79-9360-844d76233ac3/Relazione-finale-pavoncella-2011.pdf?MOD=AJPERES\&CACHEID= ROOTWORKSPACE-6c04d1f4-c914-4e79-9360-844d76233ac3-1F7b7YP (accessed on 21 September 2021).

133. Orioli, V.; Caffi, A.; Marchetto, F.; Dondina, O.; Bani, L. Quantitative selection of focal birds and mammals in higher-tier risk assessment: An application to rice cultivations. Integr. Environ. Assess. Manag. 2021, in press. [CrossRef] [PubMed]

134. Ciebiera, O.; Czechowski, P.; Morelli, F.; Piekarski, R.; Bocheński, M.; Chachulska-Serweta, J.; Jerzak, L. Selection of Urbanized Areas by Magpie Pica pica in a Medium Size City in Poland. Animals. 2021, 11, 1738. [CrossRef] [PubMed]

135. Jokimäki, J.; Suhonen, J.; Vuorisalo, T.; Kövér, L.; Kaisanlahti-Jokimäki, M.-L. Urbanization and nest-site selection of the Blackbilled Magpie (Pica pica) populations in two Finnish cities: From a persecuted species to an urban exploiter. Landsc. Urban Plan. 2017, 157, 577-585. [CrossRef]

136. IUCN. IUCN Red List Categories and Criteria: Version 3.1, 2nd ed.; IUCN: Gland, Switzerland; Cambridge, UK, 2012. Available online: https:/ / portals.iucn.org/library/sites/library/files/documents/RL-2001-001-2nd.pdf (accessed on 16 November 2021).

137. Rondinini, C.; Battistoni, A.; Peronace, V.; Teofili, C. (compilatori). Lista Rossa IUCN dei Vertebrati Italiani. Comitato Italiano IUCN e Ministero dell'Ambiente e della Tutela del Territorio e del Mare, Roma. 2013. Available online: http://www.iucn.it/listerosse-italiane.php (accessed on 17 November 2021).

138. Vickery, J.A.; Ewing, S.R.; Smith, K.W.; Pain, D.J.; Bairlein, F.; Škorpilová, J.; Gregory, R.D. The decline of Afro-Palaearctic migrants and an assessment of potential causes. IBIS 2014, 156, 1-22. [CrossRef]

139. Bowler, D.; Richter, R.L.; Eskildsen, D.; Kamp, J.; Moshøj, C.M.; Reif, J.; Strebel, N.; Trautmann, S.; Voříšek, P. Geographic variation in the population trends of common breeding birds across central Europe. Basic Appl. Ecol. 2021, 56, 72-84. [CrossRef]

140. Kuresoo, A.; Pehlak, H.; Nellis, R. Population trends of common birds in Estonia in 1983-2010. Est. J. Ecol. 2011, 60, 88-110. [CrossRef]

141. Cresswell, W.R.L.; Wilson, J.M.; Vickery, J.; Jones, P.; Holt, S. Changes in densities of Sahelian bird species in response to recent habitat degradation. Ostrich 2007, 78, 247-253. [CrossRef]

142. Ockendon, N.; Hewson, C.M.; Johnston, A.; Atkinson, P.W. Declines in British-breeding populations of Afro-Palaearctic migrant birds are linked to bioclimatic wintering zone in Africa, possibly via constraints on arrival time advancement. Bird Study 2012, 59, 111-125. [CrossRef]

143. Morrison, C.A.; Robinson, R.A.; Clark, J.A.; Risely, K.; Gill, J.A. Recent population declines in Afro-Palaearctic migratory birds: The influence of breeding and non-breeding seasons. Divers. Distrib. 2013, 19, 1051-1058. [CrossRef]

144. Howard, C.; Stephens, P.A.; Pearce-Higgins, J.W.; Gregory, R.D.; Butchart, S.H.M.; Willis, S.G. Disentangling the relative roles of climate and land cover change in driving the long-term population trends of European migratory birds. Divers. Distrib. 2020, 26, 1442-1455. [CrossRef]

145. Telenský, T.; Klvaňa, P.; Jelínek, M.; Cepák, J.; Reif, J. The influence of climate variability on demographic rates of avian Afro-palearctic migrants. Sci. Rep. 2020, 10, 17592. [CrossRef]

146. Ambrosini, R.; Rubolini, D.; Møller, A.P.; Bani, L.; Clark, J.; Karcza, Z.; Vangeluwe, D.; Du Feu, C.; Spina, F.; Saino, N. Climate change and the long-term northward shift in the African wintering range of the barn swallow Hirundo rustica. Clim. Res. 2011, 49, 131-141. [CrossRef]

147. Crawford, R.J.M.; Makhado, A.B.; Waller, L.J.; Whittington, P.A. Winners and losers-Responses to recent environmental change by South African seabirds that compete with purse-seine fisheries for food. Ostrich 2014, 85, 111-117. [CrossRef]

148. Sæther, B.-E.; Bakke, $\varnothing$. Avian life history variation and contribution of demographic traits to the population growth rate. Ecology 2000, 81, 642-653. [CrossRef]

149. Arcese, P.; Smith, J.N.M.; Hochachka, W.M.; Rogers, C.M.; Ludwig, D. Stability, regulation, and the determination of abundance in an insular song sparrow population. Ecology 1992, 73, 805-822. [CrossRef]

150. Liu, J.; Yan, H.; Li, G.; Li, S. Nest concealment is associated with reproductive traits across sympatric bird species. Ecol. Evol. 2021, 11, 14079-14087. [CrossRef]

151. Li, S.; Lu, X. Reproductive ecology of isabelline wheatears at the extreme of their altitude distribution. Ardeola 2012, 59, 301-307. [CrossRef]

152. Martin, T.E. A new view of avian life-history evolution tested on an incubation paradox. Proc. R. Soc. B Biol. Sci. 2002, 269, 309-316. [CrossRef]

153. Butler, S.J.; Gillings, S. Quantifying the effects of habitat structure on prey detectability and accessibility to farmland birds. IBIS 2004, 146, 123-130. [CrossRef]

154. Faria, N.; Morales, M.B.; Rabaça, J.E. Exploring nest destruction and bird mortality in mown Mediterranean dry grasslands: An increasing threat to grassland bird conservation. Eur. J. Wildl. Res. 2016, 62, 663-671. [CrossRef]

155. Ponce, C.; Salgado, I.; Bravo, C.; Gutiérrez, N.; Alonso, J.C. Effects of farming practices on nesting success of steppe birds in dry cereal farmland. Eur. J. Wildl. Res. 2018, 64, 13. [CrossRef] 
156. Leather, S.R. "Ecological Armageddon"-more evidence for the drastic decline in insect numbers. Ann. Appl. Biol. 2018, 172, 1-3. [CrossRef]

157. Hallmann, C.A.; Sorg, M.; Jongejans, E.; Siepel, H.; Hofland, N.; Schwan, H.; Stenmans, W.; Müller, A.; Sumser, H.; Hörren, T.; et al. More than 75 percent decline over 27 years in total flying insect biomass in protected areas. PLoS ONE 2017, 12, e0185809. [CrossRef] [PubMed]

158. Valtonen, A.; Hirka, A.; Szőcs, L.; Ayres, M.P.; Roininen, H.; Csóka, G. Long-term species loss and homogenization of moth communities in Central Europe. J. Anim. Ecol. 2017, 86, 730-738. [CrossRef] [PubMed]

159. Sánchez-Bayo, F.; Wyckhuys, K.A.G. Worldwide decline of the entomofauna: A review of its drivers. Biol. Conserv. 2019, 232, 8-27. [CrossRef]

160. Reif, J.; Hanzelka, J. Continent-wide gradients in open-habitat insectivorous bird declines track spatial patterns in agricultural intensity across Europe. Glob. Ecol. Biogeogr. 2020, 29, 1988-2013. [CrossRef]

161. Newton, I. The recent declines of farmland bird populations in Britain: An appraisal of causal factors and conservation actions. IBIS 2004, 146, 579-600. [CrossRef]

162. Eng, M.L.; Stutchbury, B.J.M.; Morrissey, C.A. Imidacloprid and chlorpyrifos insecticides impair migratory ability in a seed-eating songbird. Sci. Rep. 2017, 7, 15176. [CrossRef] [PubMed]

163. Söderström, B.; Pärt, T.; Rydén, J. Different nest predator faunas and nest predation risk on ground and shrub nests at forest ecotones: An experiment and a review. Oecologia 1998, 117, 108-118. [CrossRef] [PubMed]

164. Whittingham, M.J.; Evans, K.L. The effects of habitat structure on predation risk of birds in agricultural landscapes. IBIS 2004, 146, 210-220. [CrossRef]

165. Donald, P.F.; Sanderson, F.J.; Burfield, I.J.; van Bommel, F.P.J. Further evidence of continent-wide impacts of agricultural intensification on European farmland birds, 1990-2000. Agric. Ecosyst. Environ. 2006, 116, 189-196. [CrossRef]

166. ISTAT. 2021. Available online: http:/ / dati.istat.it/Index.aspx?QueryId=33654 (accessed on 1 October 2021).

167. Gregory, R.D.; Willis, S.G.; Jiguet, F.; Vořǐšek, P.; Klvaňová, A.; van Strien, A.; Huntley, B.; Collingham, Y.C.; Couvet, D.; Green, R.E. An indicator of the impact of climatic change on European bird populations. PLoS ONE 2009, 4, e0004678. [CrossRef]

168. Reif, J.; Št'Astný, K.; Bejček, V. Contrasting effects of climatic and habitat changes on birds with northern range limits in Central Europe as revealed by an analysis of breeding bird distribution in the Czech Republic. Acta Ornithol. 2010, 45, 83-90. [CrossRef]

169. Stiels, D.; Bastian, H.-V.; Bastian, A.; Schidelko, K.; Engler, J.O. An iconic messenger of climate change? Predicting the range dynamics of the European Bee-eater (Merops apiaster). J. Ornithol. 2021, 162, 631-644. [CrossRef]

170. Ram, D.; Axelsson, A.-L.; Green, M.; Smith, H.G.; Lindström, Å. What drives current population trends in forest birds—forest quantity, quality or climate? A large-scale analysis from northern Europe. For. Ecol. Manag. 2017, 385, 177-188. [CrossRef]

171. Griesser, M.; Lagerberg, S. Long-term effects of forest management on territory occupancy and breeding success of an open-nesting boreal bird species, the Siberian jay. For. Ecol. Manag. 2012, 271, 58-64. [CrossRef]

172. Wade, A.S.I.; Barov, B.; Burfield, I.J.; Gregory, R.D.; Norris, K.; Butler, S.J. Quantifying the Detrimental Impacts of Land-Use and Management Change on European Forest Bird Populations. PLoS ONE 2013, 8, e0064552. [CrossRef]

173. Eggers, S.; Low, M. Differential demographic responses of sympatric Parids to vegetation management in boreal forest. For. Ecol. Manag. 2014, 319, 169-175. [CrossRef]

174. Vatka, E.; Kangas, K.; Orell, M.; Lampila, S.; Nikula, A.; Nivala, V. Nest site selection of a primary hole-nesting passerine reveals means to developing sustainable forestry. J. Avian Biol. 2014, 45, 187-196. [CrossRef]

175. Lindström, A.; Svensson, S.; Green, M.; Ottvall, R. Distribution and population changes of two subspecies of Chiffchaff Phylloscopus collybita in Sweden. Ornis Svec. 2007, 17, 137-147.

176. Lehikoinen, A.; Foppen, R.P.B.; Heldbjerg, H.; Lindström, Å.; van Manen, W.; Piirainen, S.; van Turnhout, C.A.M.; Butchart, S.H.M. Large-scale climatic drivers of regional winter bird population trends. Divers. Distrib. 2016, 22, 1163-1173. [CrossRef]

177. Lehikoinen, A.; Green, M.; Husby, M.; Kålås, J.A.; Lindström, A. Common montane birds are declining in northern Europe. J. Avian Biol. 2014, 45, 3-14. [CrossRef]

178. Lehikoinen, A.; Brotons, L.; Calladine, J.; Campedelli, T.; Escandell, V.; Flousek, J.; Grueneberg, C.; Haas, F.; Harris, S.; Herrando, S.; et al. Declining population trends of European mountain birds. Glob. Chang. Biol. 2019, 25, 577-588. [CrossRef] [PubMed]

179. Le Viol, I.; Jiguet, F.; Brotons, L.; Herrando, S.; Lindström, Å.; Pearce-Higgins, J.W.; Reif, J.; Van Turnhout, C.; Devictor, V. More and more generalists: Two decades of changes in the European avifauna. Biol. Lett. 2012, 8, 780-782. [CrossRef]

180. Dorazio, R.M. Accounting for imperfect detection and survey bias in statistical analysis of presence-only data. Glob. Ecol. Biogeogr. 2014, 23, 1472-1484. [CrossRef]

181. Guillera-Arroita, G. Modelling of species distributions, range dynamics and communities under imperfect detection: Advances, challenges and opportunities. Ecography 2017, 40, 281-295. [CrossRef]

182. Fink, D.; Auer, T.; Johnston, A.; Ruiz-Gutierrez, V.; Hochachka, W.M.; Kelling, S. Modeling avian full annual cycle distribution and population trends with citizen science data. Ecol. Appl. 2020, 30, e02056. [CrossRef]

183. Royle, J.A.; Dorazio, R.M. Hierarchical Modeling and Inference in Ecology: The Analysis of Data from Populations, Metapopulations and Communities; Academic Press: San Diego, CA, USA, 2008.

184. Royle, J.A. N-Mixture Models for Estimating Population Size from Spatially Replicated Counts. Biometrics 2004, 60, 108-115. [CrossRef] [PubMed] 